\title{
GLOBAL INVERTIBILITY FOR ORIENTATION-PRESERVING SOBOLEV MAPS VIA INVERTIBILITY ON OR NEAR THE BOUNDARY
}

\author{
STEFAN KRÖMER
}

\begin{abstract}
By a result of John Ball (1981), a locally orientation preserving Sobolev map is almost everywhere globally invertible whenever its boundary values admit a homeomorphic extension. As shown here for any dimension, the conclusions of Ball's theorem and related results can be reached while completely avoiding the problem of homeomorphic extension. For suitable domains, it is enough to know that the trace is invertible on the boundary or can be uniformly approximated by such maps. An application in Nonlinear Elasticity is the existence of homeomorphic minimizers with finite distortion whose boundary values are not fixed. As a tool in the proofs, strictly orientation-preserving maps and their global invertibility properties are studied from a purely topological point of view.
\end{abstract}

Topological degree, Nonlinear Elasticity, global invertibility, approximate invertibility on the boundary, orientation-preserving deformations

\section{INTRODUCTION}

A classical problem in Nonlinear Elasticity is to determine whether a Sobolev map $y: \Omega \rightarrow \mathbb{R}^{d}$ on a bounded domain $\Omega \subset \mathbb{R}^{d}$, is invertible in a suitable sense. In this context, the map $y$ describes the deformation of an elastic solid occupying $\Omega$ in its undeformed state. In this model, lack of invertibilty corresponds to self-interpenetration which is clearly undesirable. If we assume the existence of a stored energy density (i.e., elastic deformation does not dissipate energy) with suitable properties, then a stable deformed state can be found via global minimization [4]. The typical examples for such energies enforce $y \in W^{1, p}\left(\Omega ; \mathbb{R}^{d}\right)$, strictly orientation-preserving in the sense that $\operatorname{det} \nabla y>0$ a.e. in $\Omega$.

However, even if $y \in C^{1}$ and $\operatorname{det} \nabla y$ is positive everywhere, this does not suffice to guarantee global invertibility, because different ends of the body can still overlap. The variational theory is compatible with imposing global invertibility (in a weak a.e. sense) as a constraint, the Ciarlet-Nečas condition (CNc) [8] (see Def. 2.14).

In case of a strictly orientation-preserving map, if we also assume that its boundary values match those of a homeomorphism, (CNC) always holds as a byproduct of a result of Ball [3]. The result also provides

Date: January 10, 2020. 
further topological properties of the deformation and its image. For similar purposes, this assumption also appears in [44] and other works, in particular in context of maps of finite distortion [17] (e.g.). The caveat here is that for $d \geq 3$, a homeomorphic extension can fail to exist. This issue is discussed in detail in Appendix A.

We will see that for the purpose of proving the result of [3], it suffices to know that the deformation is continuous and invertible on the boundary, or, more generally, approximately invertible on the boundary (AIB) with respect to uniform convergence (Theorem 6.1). The result requires an assumption on the topological nature of the domain, namely, that $\mathbb{R}^{d} \backslash \partial \Omega$ consists of exactly two connected components. As illustrated by examples in Appendix B, this restriction is not just a technical issue. The class AIB can also be used instead of (CNc) to implement a global invertibility constraint in Nonlinear Elasticity, because it is stable under weak convergence in suitable Sobolev spaces and therefore compatible with direct methods in the Calculus of Variations (Section 2).

A first, crucial step to connect global invertibility with invertibility on the boundary amounts to calculating the topological degree of $y$ (Theorem 4.2 in Section (4). It is of course not surprising that information on the boundary suffices for that, since the degree only depends on boundary values. Actually, if we assume in addition that $y(\bar{\Omega})=\bar{\Omega}$ and $y(\partial \Omega)=\partial \Omega$, then we are working in a class of maps with a semigroup structure, and it is well known that homeomorphisms in such a class have a degree of \pm 1 , due to a standard multiplicativity property of the degree. The main global assumption of [3], the existence of a homeomorphic extension, would allow us to reduce our more general situation to this scenario.

However, in general there is no natural group structure we could use directly. Even if there exists a degree for endomorphisms of $y(\partial \Omega)$ (neither $\partial \Omega$ nor $y(\partial \Omega)$ are always topological $(d-1)$-manifolds in our setting!), we still have the problem of linking it with the definition of the degree for continuous maps $\mathbb{R}^{d} \rightarrow \mathbb{R}^{d}$. This is not trivial, as any comparison of the two and their necessary normalizing conditions (like $\operatorname{deg}(\mathrm{id} ; \Omega ; \cdot)=1$ in $\Omega$ ) already requires a continuous map $\partial \Omega \rightarrow y(\partial \Omega)$ with a continuous extension such that both degrees are known, to act as reference map which is meaningful in both worlds. Instead, the proof of Theorem 4.2 exclusively works with the degree for continuous endomorphisms of $\mathbb{R}^{d}$ on domains. It relies on a generalized version of the Jordan-Brouwer separation theorem and a general formula for the degree of composite functions, the multiplication theorem (see [11], e.g.). 
As a second tool for the proof of Theorem 6.1 and further applications concerning global invertibility in $W^{1, p}$ for $p \geq d$ (Section [6), we develop a self-contained, purely topological theory of strictly orientation preserving maps with the help of Brouwer's degree (Section 5). Their interplay with a global invertibility constraint stated in terms of the degree is summarized in Theorem 5.10 and Corollary 5.12. Besides the generalization of [3], this can be used to complement results of [42] and [36, 37] for maps of finite distortion to prove that suitable deformations are actually homeomorphisms (Theorem 6.8 and Theorem 6.10). As a direct application, we show the existence of homeomorphic minimizers for nonlinear elastic energy functionals controlling the inner or outer distortion (Subsection 6.3), without fixing the boundary values of admissible deformations (in the spirit of [3], as in [17], e.g.) or prescribing a given Lipschitz domain as their image (as in [31]).

Another application will be presented in the forthcoming paper [23], in context of the numerical approximation of global invertibility constraints via penalty terms in the energy. One such approach is given in [24, where the penalty term acts on the full domain. However, reflecting the nonlocal nature of global invertibility, any suitable penalty term is necessarily nonlocal, and the associated computational cost can be significantly reduced by using a variant acting only on the boundary [23, thereby reducing the effective dimension of the problem. A priori, such a boundary penalty term can only hope to ensure invertibility on the boundary, which is why we need the results developed here to understand the link to full invertibility.

All results presented here apply in particular for $d=3$.

\section{Contents}

1. Introduction

1.1. Basic notation and terminology

2. Constraints related to global invertibility

2.1. Approximate invertibility

2.2. The Ciarlet-Nečas condition and condition (INV)

2.3. Maps of topological degree at most one

3. The degree: basic notation and properties

4. The degree and approximate invertibility

5. The degree and orientation preserving maps

5.1. Strictly orientation preserving maps

5.2. Strictly orientation preserving maps of degree one

6. Global invertibility in $W^{1, p}$

6.1. Ball's global invertibility revisited 
6.2. Improved invertibility exploiting finite distortion 29

6.3. Existence of homeomorphic minimizers 32

Appendix A. The problem of homeomorphic extension 35

A.1. Homeomorphic extension versus Schoenflies extension 35

A.2. Known results and counterexamples 37

A.3. Homeomorphic extension for $C^{1}$ functions on Lipschitz domains

38

Appendix B. Counterexamples for domains in with holes 40

Acknowledgements 41

References 41

1.1. Basic notation and terminology. Throughout the article, we use $p \geq d \geq 2$, where $p \in \mathbb{R}$ and $d \in \mathbb{N}$, and the following subsets of the Sobolev space $W^{1, p}\left(\Omega ; \mathbb{R}^{d}\right)$ of functions on an open set $\Omega \subset \mathbb{R}^{d}$ with values in $\mathbb{R}^{d}$ :

$$
\begin{aligned}
& W_{+}^{1, p}\left(\Omega ; \mathbb{R}^{d}\right):=\left\{y \in W^{1, p}\left(\Omega ; \mathbb{R}^{d}\right) \mid \operatorname{det} \nabla y>0 \text { a.e. in } \Omega\right\}, \\
& W_{+, \text {loc }}^{1, p}\left(\Omega ; \mathbb{R}^{d}\right):=\left\{y \in W_{\text {loc }}^{1, p}\left(\Omega ; \mathbb{R}^{d}\right) \mid \operatorname{det} \nabla y>0 \text { a.e. in } \Omega\right\} .
\end{aligned}
$$

Here, $\Omega$ is called a domain if is open and connected, and "a.e." abbreviates almost everywhere, which is understood with respect to the Lebesgue measure $\mathcal{L}^{d}$ in $\mathbb{R}^{d}$ unless specified otherwise. For any set $A \subset \mathbb{R}^{d}, \bar{A}$ is its closure and int $A$ its interior, and for $A_{1}, A_{2} \subset \mathbb{R}^{d}$, $A_{1} \subset \subset A_{2}$ means that $\bar{A}_{1}$ is compact and $\bar{A}_{1} \subset A_{2}$. An Euclidean norm is always denoted by $|\cdot|$, in any finite-dimensional real vector space that should be clear from the context, and for a point $x$ and a set $S$ in such a space, $\operatorname{dist}(x ; S):=\inf \{|x-s| \mid s \in S\}$.

Notation concerning the topological degree is introduced in Section 3.

\section{Constraints Related to GLOBal inVERTiBility}

In this section, we collect various conditions related to global invertibility that are viable as constraints for variational approaches in Nonlinear Elasticity. It is interesting to note that even in the "simple" case of $p>d$ on smooth domains, it is not clear whether the notions based on approximate invertibility coincide with or are stronger than the more classical constraints like the Ciarlet-Nečas condition, cf. Remark 2.19.

\subsection{Approximate invertibility.}

Definition 2.1 (AI: approximately invertible on a compact set). Let $K \subset \mathbb{R}^{d}$ be bounded. A continuous function $y: K \rightarrow \mathbb{R}^{d}$ is called approximately invertible on $K$ if there exists a sequence of injective 
maps $\varphi_{k} \in C\left(K ; \mathbb{R}^{d}\right)$ with $\varphi_{k} \rightarrow y$ uniformly on $K$. The class of all such maps $y$ in $C\left(K ; \mathbb{R}^{d}\right)$ is denoted by $\mathrm{AI}(K)$.

The most important examples are $K=\bar{\Omega}$ and $K=\partial \Omega$ on a domain $\Omega \subset \mathbb{R}^{d}$. As a matter of fact, the class $\operatorname{AI}(K)$ is linked to monotone mappings in the topological sense. For these, in dimension $d=2$ (but not for $d \geq 3$ ), a quite comprehensive and satisfactory theory is available [19, 20]. In this article, we are especially interested in $d \geq 3$ and $K=\partial \Omega$ :

Definition 2.2 (AIB: approximately invertible on the boundary).

Let $\Omega \subset \mathbb{R}^{d}$ be open and bounded, and $y: \bar{\Omega} \rightarrow \mathbb{R}^{d}$ with $y \in C\left(\partial \Omega ; \mathbb{R}^{d}\right)$. We say that $y$ is approximately invertible on the boundary, if $y \in$ AIB $:=$ $\operatorname{AI}(\partial \Omega)$.

From the point of view of Nonlinear Elasticity, AIB describes the class of deformations whose deformed boundaries can be moved out of selfcontact.

It is easy to see that AI is sequentially closed under uniform convergence on $K$. In suitable Sobolev spaces, this implies stability under weak convergence:

Lemma 2.3. Let $y \in W^{1, p}\left(\Omega ; \mathbb{R}^{d}\right)$ and let $\left(y_{k}\right) \subset W^{1, p}\left(\Omega ; \mathbb{R}^{d}\right)$ be a sequence with $y_{k} \rightarrow y$ weakly in $W^{1, p}$. In addition, let $K \subset \bar{\Omega}$ be compact and assume that $\left(y_{k}\right) \subset \mathcal{A} \cap \mathrm{AI}(K)$ for a set $\mathcal{A} \subset W^{1, p}\left(\Omega ; \mathbb{R}^{d}\right)$ compactly embedded in $C\left(K ; \mathbb{R}^{d}\right)$. Then $y \in \mathrm{AI}(K)$.

Proof. This follows from a straightforward diagonal subsequence argument in $C\left(K ; \mathbb{R}^{d}\right)$.

Remark 2.4. The assumptions of Lemma 2.3 are satisfied in each of the following cases:

(a) $p>d, K \subset \Omega$ and $\mathcal{A}=W^{1, p}$;

(b) $p>d, \Omega$ is a Lipschitz domain, $K \subset \bar{\Omega}$ and $\mathcal{A}=W^{1, p}$;

(c) $p \geq d, K \subset \Omega$ and $\mathcal{A}=W_{+}^{1, p}=W^{1, p} \cap\{\operatorname{det} \nabla y>0$ a.e. $\}$.

(d) $p>d-1, \mathcal{A}=\{y\} \cup\left\{y_{k} \mid k \in \mathbb{N}\right\}$ and $K \subset \Omega$ s.t. $\mathcal{A}$ is embedded and closed in $C\left(K ; \mathbb{R}^{d}\right)$.

Here, (a) and (b) are due to standard compact embeddings of $W^{1, p}$. For (c) see Remark 6.4. In (d), one has to be careful to correctly interpret $\mathcal{A}$ as a subset of $C\left(K ; \mathbb{R}^{d}\right)$ in a way independent of the choice of representatives. For instance, if we fix $x_{0} \in \Omega$, then $K_{r}:=\partial B_{r}\left(x_{0}\right)$ is admissible in $(\mathrm{d})$ for $\mathcal{L}^{1}$-a.e. $r \in\left(0\right.$, dist $\left.\left(x_{0} ; \partial \Omega\right)\right)$, essentially because we work with countably many functions and for a.e. $r, y_{k} \rightarrow y$ weakly in $W^{1, p}\left(K_{r} ; \mathbb{R}^{d} ; \mathcal{H}^{d-1}\right)$ (the Sobolev space with respect to the surface measure $\left.\mathcal{H}^{d-1}\right)$. For more details see, e.g., [33]. 
As a consequence, we can easily obtain the existence of minimizers in $\mathrm{AI}(K)$, for instance for the nonlinear elastic energies with polyconvex energy density studied by Ball [4] and Müller [32]:

Theorem 2.5. Let $p \geq d$, let $\Omega \subset \mathbb{R}^{d}$ be a Lipschitz domain, let $E$ : $W^{1, p}\left(\Omega ; \mathbb{R}^{d}\right) \rightarrow \mathbb{R} \cup\{+\infty\}$ such that $E(y)=+\infty$ for all $y \notin W_{+}^{1, p}$, i.e., whenever det $\nabla y \leq 0$ on a set of positive measure. In addition, assume that $E$ is coercive and weakly sequentially lower semincontinuous in $W^{1, p}$. If $E \not \equiv+\infty$ and $K \subset \Omega$ is compact, then $E$ attains its minimum on $\mathcal{Y}:=\mathrm{AI}(K) \cap W_{+}^{1, p}\left(\Omega ; \mathbb{R}^{d}\right)$. Moreover, if $p>d$, the above also holds for compact $K \subset \bar{\Omega}$.

Proof. This follows by the direct method.

Remark 2.6. Here and in the rest of the article, it is implicitly understood that we always use the continuous representative of $y$ if available.

All functions which admit homeomorphic extensions from the boundary into the domain are in AIB:

Proposition 2.7. Let $\Omega \subset \mathbb{R}^{d}$ be a bounded Lipschitz domain. If $y: \bar{\Omega} \rightarrow \mathbb{R}^{d}$ is continuous, and $\left.y\right|_{\Omega}: \Omega \rightarrow y(\Omega)$ is invertible, then $y \in$ AIB.

Proof. Since $\Omega$ is a Lipschitz domain, there exists a sequence of invertible maps $\Psi_{k}: \bar{\Omega} \rightarrow \Psi_{k}(\bar{\Omega}) \subset \mathbb{R}^{d}$ of class $C^{\infty}$ such that $\Psi_{k}(\Omega) \subset \subset \Omega$ (slightly smaller), while $\Psi_{k} \rightarrow$ id in $W^{1, \infty}$ as $k \rightarrow \infty$. Locally, in each cube where the boundary is represented as the graph of a Lipschitz function, $\Psi_{k}$ can be defined as the affine map slightly shrinking the local piece of $\Omega$ "down" into itself, and since all these maps are still close to the identity, they can be easily glued by a smooth decomposition of unity. Thus, $\varphi_{k}:=\left.y \circ \Psi_{k}\right|_{\partial \Omega}$ is a sequence of continuous, injective maps with $\left.\varphi_{k} \rightarrow y\right|_{\partial \Omega}$ in $C\left(\partial \Omega ; \mathbb{R}^{d}\right)$.

Remark 2.8. The converse of Proposition 2.7 includes the problem of homeomorphic extension as a special case, namely, if we only consider $y \in$ AIB which is already invertible on $\partial \Omega$. In general, it is false for $d \geq 3$ because not all such $y$ admit a homeomorphic extension into the domain, not even if $\left.y\right|_{\partial \Omega}$ is bi-Lipschitz (see Remark A.7).

We can also slightly modifiy the definition of AIB, allowing the approximants to be defined on sets approaching $\partial \Omega$ from the inside. Monotone coverings of $\Omega$ from the inside with a mild regularity property (to be used later) are helpful for that purpose:

Definition 2.9 (regular inner covering). Let $\Omega \subset \mathbb{R}^{d}$ be open. We call a family of sets $\left(\Omega_{m}\right)_{m \in \mathbb{N}}$ a regular inner covering of $\Omega$, if $\Omega_{m}$ is open and bounded, $\Omega_{m} \subset \Omega_{m+1} \subset \subset \Omega, \bigcup_{m \in \mathbb{N}} \Omega_{m}=\Omega$ and $\mathcal{L}^{d}\left(\partial \Omega_{m}\right)=0$ for every $m \in \mathbb{N}$. 
Remark 2.10. It is not difficult to see that a regular inner covering always exists; as a matter of fact, we could even assume that $\partial \Omega_{m}$ is of class $C^{\infty}$ instead of just being a set of measure zero. Moreover, if we know that $\mathbb{R}^{d} \backslash \partial \Omega$ has only two connected components (which is important to apply Theorem 4.2 below), we can always find a regular inner covering such that all $\Omega_{m}$ inherit this property.

The following variants of AIB and AI are particularly useful when $y$ is continuous in $\Omega$ but cannot be continuously extended to $\partial \Omega$ (like maps in $W_{+}^{1, p}$ with $\left.p=d\right)$ :

Definition $2.11\left(\mathrm{AIB}_{\text {loc }}, \mathrm{AI}_{\text {loc }}(\Omega)\right)$. Let $\Omega \subset \mathbb{R}^{d}$ be open, let $y: \Omega \rightarrow$ $\mathbb{R}^{d}$ be continuous and let $\left(\Omega_{m}\right)_{m \in \mathbb{N}}$ be regular inner covering of $\Omega$. We say that $y \in \mathrm{AIB}_{\text {loc }}$ with respect to $\left(\Omega_{m}\right)$, if for each $m \in \mathbb{N}, y \in \mathrm{AIB}$ on $\Omega_{m}$, i.e., if there exists continuous and injective maps $\varphi_{k}^{(m)}: \partial \Omega_{m} \rightarrow \mathbb{R}^{d}$ such that

$$
\left\|y-\varphi_{k}^{(m)}\right\|_{C\left(\partial \Omega_{m}\right)} \rightarrow 0 \quad \text { as } k \rightarrow \infty .
$$

Analogously, we say that $y \in \mathrm{AI}_{\text {loc }}(\Omega)$ with respect to $\left(\Omega_{m}\right)$, if for each $m \in \mathbb{N}, y \in \operatorname{AI}\left(\Omega_{m}\right)$.

Remark 2.12. It is easy to see that both Lemma 2.3 and Theorem 2.5 also hold for $\mathrm{AIB}_{\text {loc }}$ or $\mathrm{AI}_{\text {loc }}(\Omega)$ instead of $\mathrm{AI}(K)$. In fact, we do not even use that $\left(\Omega_{m}\right)$ is a countable family, because there are no conditions linking $\varphi_{k}^{(m)}$ for two different values of $m$. This means that diagonal subsequences as in the proof of Lemma 2.3 can be chosen for each fixed $m$ separately, by the axiom of choice if we have more than countably many $m$.

Remark 2.13. $\mathrm{AIB}_{\text {loc }}$ can potentially still be used in settings with low regularity like $W^{1, p}$ with $d-1<p<d$, cf. Remark 2.4. By contrast, this does not work for $\mathrm{AI}_{\text {loc }}(\Omega)$ as defined here. However, approximate invertibility defined with respect to weak convergence in the Sobolev space is a still meaningful concept [6].

An explicit example for an existence results in the spirit of Theorem 2.5 using either $\mathrm{AIB}_{\text {loc }}$ or $\mathrm{AI}_{\text {loc }}(\Omega)$ as a constraint is given in Subsection 6.3. Unlike all the other invertibility constraints presented in this section, $\mathrm{AIB}$ and $\mathrm{AIB}_{\text {loc }}$ only restrict the global behavior of $y$ with information given on or near the boundary, but not its local properties inside the domain. However, in $W_{+}^{1, p}$ with $p \geq d$, local restrictions still follow automatically, see Remark 2.19. Practically, AIB can be easier to show for a given deformation, though.

2.2. The Ciarlet-Nečas condition and condition (INV). For comparison, we briefly recall two invertibility constraints often used in the literature.

The standard constraint for $p>d$ was introduced in [8]: 
Definition 2.14 (Ciarlet-Nečas condition (

Let $\Omega \subset \mathbb{R}^{d}$ be open and bounded. A map $y \in W_{+}^{1, p}\left(\Omega ; \mathbb{R}^{d}\right), p \geq d$, satisfies the Ciarlet-Nečas condition, or, shortly, $y \in \mathrm{CNC}$, if

$$
\int_{\Omega} \operatorname{det} \nabla y(x) d x \leq \mathcal{L}^{d}(y(\Omega)) .
$$

Using the area formula as in the proof of Lemma 2.15 below, it is not difficult to see that (CNc) is equivalent to injectivity almost everywhere in the sense that the set of all points in $\Omega$ where $y$ fails to be injective is of measure zero.

If $p>d$ and $\Omega$ is Lipschitz, (CNC) is stable under weak convergence in $W^{1, p}$ for $p>d$ (proved as part of [8, Theorem 5]). If $p=d$, it can happen that the left hand side of (CNC) jumps down in the limit along a weakly converging sequence, due to concentration effects at the boundary (cf. [32], [21]). Nevertheless, the right hand side actually produces a matching jump in such cases because (CNc) still behaves stably as a whole along sequences in $W_{+}^{1, d}$ weakly converging in $W^{1, d}$, a result obtained in [39] in broader context. Alternatively, one can use the even more general results of [13], or Remark 2.19] (e) below.

The following lemma also used in [39] shows that there is no point in defining a "loc" version of ( $\mathrm{CNC}$. In view of all the properties known for functions in $W_{+}^{1, d}$ in the interior of the domain (cf. Remark 6.4), and the local equi-integrability result for the determinant of [32, it can also be the basis of yet another, more direct proof of the result of [39] in the special case $W_{+}^{1, d}$.

Lemma 2.15. Let $\Omega \subset \mathbb{R}^{d}$ be open and bounded, let $y \in W_{+}^{1, d}\left(\Omega ; \mathbb{R}^{d}\right)$ and let $\left(\Omega_{m}\right)_{m \in \mathbb{N}} \subset \Omega$ be a sequence of open sets with $\Omega_{m} \subset \Omega_{m+1} \subset \subset \Omega$ and $\bigcup_{m \in \mathbb{N}} \Omega_{m}=\Omega$. Then $y$ satisfies (CNC) on $\Omega$ if and only if it satisfies (CNc) on $\Omega_{m}$ for all $m$.

Proof. By the area formula (see, e.g., [11, Theorem 5.34]),

$$
\int_{\Omega}|\operatorname{det} \nabla y| d x=\int_{y(\Omega)} \# y^{-1}(\{z\}) d z,
$$

where $\# y^{-1}(\{z\})$ denotes the number of elements of $y^{-1}(\{z\})$. In view of (2.1), (CNC) can be expressed as $\# y^{-1}(\{z\})=1$ for a.e. $z$, and it is clear that (CNc) on $\Omega$ implies (CNC) on every smaller set like $\Omega_{m}$. The converse follows by monotone convergence.

Another condition implying both local and global invertibility properties was developed in [33], mainly intended for settings involving cavitation in $W^{1, p}$ with $d-1<p<d$. It uses the fact that the degree only depends on boundary values, cf. Section 3 , and the concept of the topological image $\operatorname{im}_{T}$ based on the degree, cf. Lemma 5.5. 
Definition 2.16 (Müller-Spector condition (INV)). Let $\Omega \subset \mathbb{R}^{d}$ be a bounded domain and $y: \Omega \rightarrow \mathbb{R}^{d}$. The map $y$ satisfies condition (INV), or, shortly, $y \in$ INV, if the following holds:

For every $a \in \Omega$, there exists a set $N_{a} \subset \mathbb{R}$ with $\mathcal{L}^{d}\left(N_{a}\right)=0$ such that $y \in C\left(\partial B_{r}(a) ; \mathbb{R}^{d}\right)$ for all $r \in(0$, dist $(a ; \partial \Omega)) \backslash N_{a}$,

(i) $y(x) \in \operatorname{im}_{T}\left(y ; B_{r}(a)\right) \cup y\left(\partial B_{r}(a)\right)$ for a.e. $x \in \bar{B}_{r}(a)$, and

(ii) $y(x) \in \mathbb{R}^{d} \backslash \operatorname{im}_{T}\left(y ; B_{r}(a)\right)$ for a.e. $x \in \Omega \backslash B_{r}(a)$.

Condition (INV) is stable under weak convergence in $W^{1, p}$ for $p>d-1$ [33. Lemma 3.3].

2.3. Maps of topological degree at most one. Another property that prevents global self-interpenetration in suitable settings can be expressed with the help of the topological degree (cf. Section 3). This turns out to be a natural common denominator of all the other global invertibility conditions, at least within $W_{+}^{1, p}$ for $p \geq d$.

Definition 2.17 (Maps of degree at most one).

Let $\Omega \subset \mathbb{R}^{d}$ be open and bounded and $y: \bar{\Omega} \rightarrow \mathbb{R}^{d}$. If $y \in C\left(\partial \Omega ; \mathbb{R}^{d}\right)$, we say that $y$ is of degree (at most) one, or, shortly, $y \in$ DEG1, if

$$
\operatorname{deg}(y ; \Omega ; z) \leq 1 \text { for all } z \in \mathbb{R}^{d} \backslash y(\partial \Omega)
$$

If $\left(\Omega_{k}\right)_{k \in \mathbb{N}}$ is a regular inner covering of $\Omega$ (see Definition 2.9), we say that $y$ is of degree (at most) one locally near the boundary, or, shortly, $y \in \mathrm{DEG}_{\mathrm{loc}}$, if

$$
\operatorname{deg}\left(y ; \Omega_{k} ; z\right) \leq 1 \quad \text { for each } k \in \mathbb{N} \text { and all } z \in \mathbb{R}^{d} \backslash y\left(\partial \Omega_{k}\right)
$$

Remark 2.18. As the degree is continuous with respect to uniform convergence on the boundary (stability), Lemma 2.3 and Theorem 2.5 also hold if we replace $\mathrm{AI}(K)$ by

(a) DEG1, if $p>d$ and $\Omega$ is Lipschitz, or

(b) DEG1 $1_{\text {loc }}$, if $p \geq d$.

In other words, DEG1 and DEG1 $1_{\text {loc }}$, too, are stable under weak convergence and viable as variational constraints.

Remark 2.19. Let $\Omega \subset \mathbb{R}^{d}$ be a bounded domain. Whenever AIB or $\mathrm{AIB}_{\text {loc }}$ are involved, we also assume that $\mathbb{R}^{d} \backslash \partial \Omega$ has only two connected components, to be able to apply Theorem 4.2. Consider the following two classes of strictly orientation preserving Sobolev maps:

$$
\begin{aligned}
& \mathcal{Y}_{+}^{p}(\Omega):=W_{+}^{1, p}\left(\Omega ; \mathbb{R}^{d}\right) \cap C\left(\Omega ; \mathbb{R}^{d}\right) \quad \text { and } \\
& \mathcal{Y}_{+}^{p}(\bar{\Omega}):=W_{+}^{1, p}\left(\Omega ; \mathbb{R}^{d}\right) \cap C\left(\bar{\Omega} ; \mathbb{R}^{d}\right) \cap\left\{y \mid \mathcal{L}^{d}(y(\partial \Omega))=0\right\}
\end{aligned}
$$


(If $p>d$ and $\Omega$ is a Lipschitz domain, $\mathcal{Y}_{+}^{p}(\Omega)=\mathcal{Y}_{+}^{p}(\bar{\Omega})=W_{+}^{1, p}\left(\Omega ; \mathbb{R}^{d}\right)$.) Within $\mathcal{Y}_{+}^{p}(\Omega)$ or $\mathcal{Y}_{+}^{p}(\bar{\Omega})$, respectively, the invertibility conditions are related as follows for $p \geq d$ :
(a) $\mathcal{Y}_{+}^{p}(\bar{\Omega}) \cap \mathrm{AIB}$
$\subset \mathcal{Y}_{+}^{p}(\bar{\Omega}) \cap$ DEG1;
(b) $\mathcal{Y}_{+}^{p}(\bar{\Omega}) \cap \mathrm{CNC}=\mathcal{Y}_{+}^{p}(\bar{\Omega}) \cap \mathrm{DEG} 1$;
(c) $\mathcal{Y}_{+}^{p}(\bar{\Omega}) \cap \mathrm{DEG}_{1 \mathrm{loc}}=\mathcal{Y}_{+}^{p}(\bar{\Omega}) \cap \mathrm{DEG} 1$;
(d) $\mathcal{Y}_{+}^{p}(\Omega) \cap \mathrm{AIB}_{\text {loc }} \subset \mathcal{Y}_{+}^{p}(\Omega) \cap \mathrm{DEG}_{1 \mathrm{loc}}$;
(e) $\mathcal{Y}_{+}^{p}(\Omega) \cap \mathrm{AI}_{\text {loc }}(\Omega) \subset \mathcal{Y}_{+}^{p}(\Omega) \cap \mathrm{DEG}_{1 \text { loc }}$;
(f) $\mathcal{Y}_{+}^{p}(\Omega) \cap \mathrm{CNC}=\mathcal{Y}_{+}^{p}(\Omega) \cap \mathrm{DEG} 1_{\mathrm{loc}}$;
(g) $\mathcal{Y}_{+}^{p}(\Omega) \cap \mathrm{INV}=\mathcal{Y}_{+}^{p}(\Omega) \cap \mathrm{DEG} 1_{\mathrm{loc}}$.

For a proof of some of these connections, we occasionally need properties of the degree (Section 3) and other results presented later. Throughout, Lemma 6.7 ensures that any $y \in W_{+}^{1, p}$ is strictly orientation preserving in the topological sense of Section 5; in particular, its degree can never be negative.

The inclusions (a) and (d) are consequences of Theorem 4.2 applied on $\Omega_{m}$ (see also Remark 2.10), and (e) analogously follows from Theorem 4.8. In case of (c), " $\subset$ " is a consequence of the continuity of the degree (stability) while " $\supset$ " follows from Corollary 5.12 (i). Since we also have Lusin's property (N) (cf. Remark 6.4), $y\left(\partial \Omega_{m}\right)$ has empty interior for all the sets of the regular inner covering of $\Omega$ associated to DEG1 $1_{\text {loc }}$. For (f), one can use Lemma 2.15 and the change-of-variables formula involving the degree (6.2) with $f=1$. To see (b), we combine (c) and (f) with Lemma 2.15, Finally, (g) is the content of Lemma 2.20 below.

In (a), (d) and (e), I do not know if the reverse inclusions hold (for $d \geq 3)$. Similarly, while we trivially have that $\operatorname{AI}(\bar{\Omega}) \subset \mathrm{AIB}$ and $\mathrm{AI}_{\text {loc }}(\Omega) \subset \operatorname{AIB}_{\text {loc }}$ (with the covering $\left(\Omega_{m}\right)$ fixed), it is not clear if equality holds (given that $\mathbb{R}^{d} \backslash \partial \Omega$ has only two connected components). This is related to a weaker variant of the problem of homeomorphic extension for which the counterexamples mentioned in Appendix A do not apply.

Lemma 2.20. Let $\Omega \subset \mathbb{R}^{d}$ be a bounded domain and $p \geq d$. Then

$$
W_{+}^{1, p}\left(\Omega ; \mathbb{R}^{d}\right) \cap C\left(\Omega ; \mathbb{R}^{d}\right) \cap \mathrm{INV}=W_{+}^{1, p}\left(\Omega ; \mathbb{R}^{d}\right) \cap C\left(\Omega ; \mathbb{R}^{d}\right) \cap \mathrm{DEG} 1_{\text {loc }} .
$$

Proof. Let $y \in W_{+}^{1, p}\left(\Omega ; \mathbb{R}^{d}\right) \cap C\left(\Omega ; \mathbb{R}^{d}\right)$. By Lemma 6.7, $y$ is strictly orientation preserving in the topological sense, and by Remark 6.4, it satisfies Lusin's property $(\mathrm{N})$. In particular, $y\left(\partial \Omega_{m}\right)$ has measure zero and thus empty interior.

"C": Suppose that $y$ also satisfies (INV). By [33, Lemma 3.4], y is invertible almost everywhere, which implies $y \in \mathrm{CNC}$ by the area 
formula (as in the proof of Lemma 2.15). By Remark 2.19 (f), we conclude that $y \in \mathrm{DEG}_{\mathrm{loc}}$.

"つ": Given $y \in \mathrm{DEG}_{\mathrm{loc}}$, Part (i) of (INV) follows from Lemma 5.5 (iii). Part (ii) follows from the fact that by Theorem 5.10 (ii) and (iii) (applied with $U=\Omega_{m}$, for all $m$ ), $y^{-1}(\{z\})$ can only have more than one connected component if all of its components touch $\partial \Omega$ (see also the Remarks 5.7 and 5.8). Here, $z \in \mathbb{R}^{d}$ is arbitrary.

\section{The DEGREE: BASIC NOTATION AND PROPERTIES}

In the next two sections, we will heavily use the topological degree (Brouwer's degree). We therefore briefly recall its main features. For a definition and its properties see for instance [22, [11] or [35].

The degree for functions in $\mathbb{R}^{d}$ is a number

$$
\operatorname{deg}(y ; A ; z) \in \mathbb{Z} \quad \text { if } z \notin y(\partial A),
$$

defined for any continuous map $y: \bar{A} \rightarrow \mathbb{R}^{d}$ on an open and bounded set $A \subset \mathbb{R}^{d}$, with respect to a value $z \in \mathbb{R}^{d}$. By the Tietze extension theorem, we can always assume that $y: \mathbb{R}^{d} \rightarrow \mathbb{R}^{d}$ is continuous. The restriction on the admissible points $z$ in (3.1) is necessary for its definition. Throughout, it is always assumed to be present, even if not stated explicitly in shorthand notations like $\operatorname{deg}(y ; A ; \cdot)$.

Besides being integer-valued, the key properties of the degree are the following:

$$
\begin{array}{ll}
\text { (nomalization) } & \operatorname{deg}(\mathrm{id} ; A ; z)=1 \text { for all } z \in A . \\
\text { (additivity) } & \operatorname{deg}\left(y ; A_{1} \cup A_{2} ; z\right)=\operatorname{deg}\left(y ; A_{1} ; z\right)+\operatorname{deg}\left(y ; A_{2} ; z\right) \\
& \text { if } A_{1} \cap A_{2}=\emptyset \text { and } z \notin \partial A_{1} \cup \partial A_{2} . \\
\text { (solvability) } & \text { If } \operatorname{deg}(y ; A ; z) \neq 0 \text { for a } z \notin y(\partial A) \\
& \operatorname{then} \text { there exists } x \in A \text { with } y(x)=z . \\
\text { (homotopy } & \operatorname{deg}\left(y_{t} ; A_{t} ; z_{t}\right)=\operatorname{deg}\left(y_{0} ; A_{0} ; z_{0}\right) \text { for all } t \in[0,1], \\
\text { invariance) } & \text { if } z_{t} \notin y_{t}\left(\partial A_{t}\right) \text { for all } t \in[0,1] \text { and }(\underline{3.2}) \text { holds. }
\end{array}
$$

Here, $y_{t}$ and $z_{t}$ are assumed to be a homotopies along $A_{t}$ in the sense that

$$
\begin{aligned}
& V:=\left\{(x, t) \mid t \in[0,1] \text { and } x \in A_{t}\right\} \\
& \text { is bounded and open relative to } \mathbb{R}^{d} \times[0,1], \\
& (t, x) \mapsto y_{t}(x), \bar{V} \rightarrow \mathbb{R}^{d}, \text { is continuous and } \\
& t \mapsto z_{t},[0,1] \rightarrow \mathbb{R}^{d}, \text { is continuous. }
\end{aligned}
$$

In many cases, homotopy invariance is only stated and applied with cylinders $V=[0,1] \times A$, which also suffices for us here. For the general version see [22] (e.g.). 
Besides solvability and additivity, we here mainly use a few other properties of the degree which can be derived from homotopy invariance:

$$
\begin{array}{lrl}
\text { (continuity) } & z \mapsto \operatorname{deg}(y ; A ; z) \text { is continuous on } \mathbb{R}^{d} \backslash y(\partial A) . \\
\text { (stability) } & \operatorname{deg}\left(y_{1} ; A ; z\right)=\operatorname{deg}\left(y_{2} ; A ; z\right) \\
& \text { if }\left\|y_{1}-y_{2}\right\|_{C\left(\partial A ; \mathbb{R}^{d}\right)}<\operatorname{dist}\left(z ; y_{1}(\partial A)\right) . \\
\text { (bnd. controlled) } & \operatorname{deg}\left(y_{1} ; A ; \cdot\right)=\operatorname{deg}\left(y_{1} ; A ; \cdot\right) \text { if } y_{1}=y_{2} \text { on } \partial A .
\end{array}
$$

Since the degree is integer-valued, continuity means it is locally constant. Stability is also a continuity property, now in $y$ instead of $z$. "Boundary controlled" means that as far as $y$ is concerned, the degree is fully determined by the values of $y$ on $\partial A$. As we can always extend continuous functions from a compact set like $\partial A$ to the whole space, the degree is well-defined also for functions that are only given and continuous on $\partial A$.

To explicitly compute the degree in some examples, the following partial representation is helpful: If $y \in C^{1}\left(\bar{A} ; \mathbb{R}^{d}\right)$ and $z$ is a regular value of $y$, i.e., $\operatorname{det} \nabla y(x) \neq 0$ for each $x \in y^{-1}(\{z\})$, then

$$
\operatorname{deg}(y ; A ; z)=\sum_{x \in y^{-1}(\{z\})} \operatorname{sgn}(\operatorname{det} \nabla y(x)) \quad \text { if } z \notin y(\partial A) .
$$

Here, $\operatorname{sgn}$ denotes the $\operatorname{sign}(\operatorname{sgn}(t)=t /|t|$ if $t \neq 0$, and $\operatorname{sgn}(0)=$ $0)$. This formula can also be used as the basis of a definition of the degree. Besides, it determines the behavior of the degree with respect to reflections, a property which generalizes to all continuous $y$ :

$$
\operatorname{deg}(R y ; A ; R z)=-\operatorname{deg}(y ; A ; z) \quad \text { if } z \notin y(\partial A) \text { and } R \text { is a reflection, }
$$

i.e., if $R \in O(d)$ with $\operatorname{det} R=-1$.

\section{The DEGREe And APPRoximate INVERTIBILITY}

Below, we repeatedly split sets in $\mathbb{R}^{d}$ into their bounded and unbounded connected components. For this purpose, we introduce the following shorthand notation.

Definition 4.1 ( $\mathcal{B}$ and $\mathcal{U}$ : bounded and unbounded components). Given a compact set $K \subset \mathbb{R}^{d}$, we decompose

$$
\mathbb{R}^{d} \backslash K=\mathcal{B}\left(\mathbb{R}^{d} \backslash K\right) \cup \mathcal{U}\left(\mathbb{R}^{d} \backslash K\right),
$$

where $\mathcal{U}\left(\mathbb{R}^{d} \backslash K\right)$ denotes the unbounded connected component of $\mathbb{R}^{d} \backslash$ $K$, and $\mathcal{B}\left(\mathbb{R}^{d} \backslash K\right)$ denotes the union of all bounded connected components of $\mathbb{R}^{d} \backslash K$. If the choice of the set $K$ is obvious from the context, we simply write $\mathcal{B}$ and $\mathcal{U}$.

A core ingredient of this article is the following statement about the degree of maps that are approximately invertible on the boundary. 
Theorem 4.2. Let $\Omega \subset \mathbb{R}^{d}$ be a bounded domain such that $\mathbb{R}^{d} \backslash \partial \Omega$ has exactly two connected components, let $y \in C\left(\bar{\Omega} ; \mathbb{R}^{d}\right) \cap \mathrm{AIB}$ and let

$$
\operatorname{im}_{T}(y ; \Omega):=\left\{z \in \mathbb{R}^{d} \backslash y(\partial \Omega) \mid \operatorname{deg}(y ; \Omega ; z) \neq 0\right\} .
$$

Then there exists a fixed $\sigma \in\{ \pm 1\}$ such that

$$
\operatorname{deg}(y ; \Omega ; z)=\sigma \quad \text { for every } z \in \operatorname{im}_{T}(y ; \Omega)
$$

Moreover, $\operatorname{im}_{T}(y ; \Omega)$ is an open subset of $\mathcal{B}\left(\mathbb{R}^{d} \backslash y(\partial \Omega)\right)$, and any connected component of $\mathcal{B}\left(\mathbb{R}^{d} \backslash y(\partial \Omega)\right)$ is either fully contained in $\operatorname{im}_{T}(y ; \Omega)$ or does not intersect $\operatorname{im}_{T}(y ; \Omega)$ at all.

Remark 4.3. Even if $y \in \operatorname{AI}(\bar{\Omega}) \subset \mathrm{AIB}$, it can happen that $\operatorname{im}_{T}(y ; \Omega)$ is not connected, for instance when $y$ compresses a surface bisecting $\Omega$ to a point.

Remark 4.4. If $\left.y\right|_{\partial \Omega}$ is already injective, it suffices to use $\varphi_{k}:=\left.y\right|_{\partial \Omega}$ to show that $y \in$ AIB. In this special case, Theorem 4.2 reduces to [34, Proposition 2.2], which follows from the proof of a generalization of the Jordan Separation Theorem (see, e.g., [11, Theorem 3.29 and its proof]). However, assuming invertibility on the boundary rules out deformations with self-contact, while Theorem 4.2 still applies with a suitable sequence $\varphi_{k}$.

Remark 4.5. The assumption that $\mathbb{R}^{d} \backslash \partial \Omega$ has only two connected components cannot be dropped, not even for orientation preserving maps. Counterexamples are given in Appendix B. Topologically speaking, this assumption expresses that $\partial \Omega$ is oriented in a degenerate sense inherited from $\Omega$ and the ambient space.

Remark 4.6. Theorem 4.2 in particular applies for every bounded Lipschitz domain $\Omega$ with connected boundary. Here and throughout the article, Lipschitz domain is understood in the strong sense, i.e., the boundary can be locally (in $\mathbb{R}^{d}$ ) represented as the graph of a Lipschitz function. In this case, $\mathbb{R}^{d} \backslash \partial \Omega$ has exactly two connected components, $\Omega$ being the bounded one, because a neighborhood of $\partial \Omega$ in $\Omega$ is connected.

Remark 4.7 (A degree on $y(\partial \Omega)$ ). If $\operatorname{im}_{T}(y ; \Omega) \neq \emptyset$, Theorem 4.2 allows us to define a degree for continuous maps $f: y(\partial \Omega) \rightarrow y(\partial \Omega)$. For that purpose, choose a point $z_{0} \in \operatorname{im}_{T}(y ; \Omega)$ (i.e., so that $\operatorname{deg}\left(y ; \Omega ; z_{0}\right)=\sigma \in$ $\{ \pm 1\})$ and a constant $\sigma_{0} \in\{ \pm 1\}$ and define

$$
\operatorname{deg}_{y(\partial \Omega)}(f):=\sigma_{0} \operatorname{deg}\left(\hat{f} \circ y ; \Omega ; z_{0}\right),
$$

where $\hat{f}: \mathbb{R}^{d} \rightarrow \mathbb{R}^{d}$ is an arbitrary continuous extension of $f$. This is well defined, since the right hand side only depends on the values of $\left.\hat{f} \circ y\right|_{\partial \Omega}=\left.f \circ y\right|_{\partial \Omega}$. The definition depends both on $y$ and on $z_{0}$. However, the dependence on $y$ (as a parametrization of $y(\partial \Omega)$ ) only enters through the orientation encoded in $\sigma$, and if we fix a connected 
component $\hat{B}$ of $\operatorname{im}_{T}(y ; \Omega)$, the definition the definition does not depend to the choice of $z_{0}$ within $\hat{B}$. In fact, we thus naturally obtain a whole family of degrees, one for each such connected component $\hat{B}$.

\section{Proof of Theorem 4.2. Let}

$$
\hat{\mathcal{U}}:=\left\{z \in \mathbb{R}^{d} \backslash y(\partial \Omega) \mid \operatorname{deg}(y ; \Omega ; z)=0\right\}=\mathbb{R}^{d} \backslash\left[y(\partial \Omega) \cup \operatorname{im}_{T}(y ; \Omega)\right]
$$

(i) First observe that by the solvability $\operatorname{property,} \operatorname{deg}(y ; \Omega ; z)=0$ for some $z \in \mathcal{U}:=\mathcal{U}\left(\mathbb{R}^{d} \backslash y(\partial(\Omega))\right.$, since there clearly exist values $z \in \mathcal{U}$ which are not in the bounded set $y(\Omega)$. As $\operatorname{deg}(y ; \Omega ; \cdot)$ is constant on connected components of $\mathbb{R}^{d} \backslash y(\partial \Omega)$ (continuity), we infer that $\mathcal{U} \subset \hat{\mathcal{U}}$. In addition, connected components of $\mathcal{U}$ are either fully contained in $\hat{\mathcal{U}}$ or do not intersect $\hat{\mathcal{U}}$ at all.

It remains to show that $\operatorname{deg}(y ; \Omega ; z)=\sigma \in\{ \pm 1\}$ for $z \in \operatorname{im}_{T}(y ; \Omega)$. By the Tietze extension theorem, both $\varphi_{k}$ and $\varphi_{k}^{-1}$ have continuous (but not necessarily invertible) extensions to $\mathbb{R}^{d}$, say, $Y_{k}$ and $Z_{k}$. Clearly,

$$
\left(Z_{k} \circ Y_{k}\right)(x)=x \quad \text { for every } x \in \partial \Omega,
$$

and since the degree only depends on the values on the boundary, this implies that

$$
\operatorname{deg}\left(Z_{k} \circ Y_{k} ; \Omega ; x\right)=\operatorname{deg}(\mathrm{id} ; \Omega ; x)= \begin{cases}1 & \text { for every } x \in \Omega, \\ 0 & \text { for every } x \in \mathbb{R}^{d} \backslash \bar{\Omega} .\end{cases}
$$

Due to our topological assumption on $\Omega$ and the Jordan Separation Theorem (see, e.g., [11, Theorem 3.29$]), \mathbb{R}^{d} \backslash \varphi_{k}(\partial \Omega)$ also has exactly two connected components, $\mathcal{B}_{k}:=\mathcal{B}\left(\mathbb{R}^{d} \backslash \varphi_{k}(\partial \Omega)\right)$ and $\mathcal{U}_{k}:=\mathcal{U}\left(\mathbb{R}^{d} \backslash\right.$ $\left.\varphi_{k}(\partial \Omega)\right)$. Moreover, $\mathcal{B}_{k} \subset Y_{k}(\Omega)$ and $\operatorname{deg}\left(Y_{k} ; \Omega ; z\right)=0$ for $z \in \mathcal{U}_{k}$. As a consequence of the multiplication formula for the degree [11, Theorem 2.10], we get that

$$
\operatorname{deg}\left(Z_{k} \circ Y_{k} ; \Omega ; x\right)=\operatorname{deg}\left(Z_{k} ; \mathcal{B}_{k} ; x\right) \operatorname{deg}\left(Y_{k} ; \Omega ; z\right) \quad \text { for } z \in \mathcal{B}_{k} .
$$

Here, notice that $\operatorname{deg}\left(Y_{k} ; \Omega ; \cdot\right)$ is constant on $\mathcal{B}_{k}$. In view of (4.1) and the fact that the degree is integer-valued, (4.2) entails that for each $k$, there exists a $\sigma_{k} \in\{ \pm 1\}$ such that

$$
\sigma_{k}=\operatorname{deg}\left(Z_{k} ; \mathcal{B}_{k} ; x\right)=\operatorname{deg}\left(Y_{k} ; \Omega ; z\right) \quad \text { for } x \in \Omega \text { and } z \in \mathcal{B}_{k} .
$$

In addition, by the stability of the degree,

$$
\begin{aligned}
& \operatorname{deg}\left(Y_{k} ; \Omega ; z\right)=\operatorname{deg}(y ; \Omega ; z) \\
& \text { for every } z \in \mathbb{R}^{d} \text { with } \operatorname{dist}(z ; y(\partial \Omega))>\left\|Y_{k}-y\right\|_{C(\partial \Omega)} .
\end{aligned}
$$

Since $\operatorname{deg}(y ; \Omega ; z)=0$ for all $z \in \hat{\mathcal{U}}$, (4.3) and (4.4) imply that

$$
\mathcal{B}_{k} \supset V_{k}:=\operatorname{im}_{T}(y ; \Omega) \cap\left\{\begin{array}{l}
z \mid \begin{array}{l}
z \in \mathcal{B}\left(\mathbb{R}^{d} \backslash y(\partial \Omega)\right) \text { and } \\
\operatorname{dist}(z ; y(\partial \Omega))>\left\|Y_{k}-y\right\|_{C(\partial \Omega)}
\end{array}
\end{array}\right\}
$$


and

$$
\sigma_{k}=\operatorname{deg}(y ; \Omega ; \cdot) \quad \text { is constant on } V_{k} \text { for each } k .
$$

Since $\left\|Y_{k}-y\right\|_{C(\partial \Omega)} \rightarrow 0$ as $k \rightarrow \infty$, the limit of the increasing sequence of open sets $V_{k}$ is given by

$$
\begin{aligned}
& \operatorname{im}_{T}(y ; \Omega)=\bigcup_{k} V_{k}=\left\{z \in \mathbb{R}^{d} \mid \begin{array}{l}
z \text { is in a bounded connected } \\
\text { component of } \mathbb{R}^{d} \backslash(y(\partial \Omega) \cup \mathcal{U})
\end{array}\right\} \\
& \subset\left\{z \in \mathbb{R}^{d} \backslash y(\partial \Omega) \mid z \in \mathcal{B}_{k} \text { for almost all } k\right\} \text {. }
\end{aligned}
$$

By (4.3) and (4.4), we also get that $\sigma_{k} \rightarrow \sigma$, whence

$$
\operatorname{deg}(y ; \Omega ; z)=\sigma \quad \text { for all } z \in \operatorname{im}_{T}(y ; \Omega) \text {, with fixed } \sigma \in\{ \pm 1\} \text {. }
$$

The remaining assertions are a straightforward consequence of the fact that $\operatorname{deg}(y ; \Omega ; \cdot)$ is constant on each connected component of $\mathbb{R}^{d} \backslash y(\partial \Omega)$.

The equivalent of Theorem 4.2 for $\operatorname{AI}(\bar{\Omega})$ is a similar but easier because the degree of full homeomorphisms is known. It works for all domains:

Theorem 4.8. Let $\Omega \subset \mathbb{R}^{d}$ be a bounded domain and let $y \in C\left(\bar{\Omega} ; \mathbb{R}^{d}\right) \cap$ $\operatorname{AI}(\bar{\Omega})$. Then all the assertions of Theorem 4.2 hold.

Proof. For the approximating homeomorphisms $\varphi_{k}: \bar{\Omega} \rightarrow \varphi_{k}(\bar{\Omega})$, it is well known that $\operatorname{deg}\left(\varphi_{k} ; \Omega ; z\right)=\sigma_{k} \in\{-1,1\}$ for every $z \notin \varphi_{k}(\partial \Omega)$ (this is a simpler application of the multiplication theorem for the degree), and that the bounded connected component of $\mathbb{R}^{d} \backslash \varphi_{k}(\partial \Omega)$ is given by $\mathcal{B}_{k}:=\varphi_{k}(\Omega)$. The rest of the proof is analogous to the proof of Theorem 4.2 .

\section{The DEGREe And orientation PRESERVing MaPS}

In this section, we study the consequences of Theorem 4.2 for the case of strictly orientation preserving maps. Some of the results in this section are essentially known (cf. [44, in particular) and the technique is classical, but there is no comprehensive collection suitable for our purposes. For this reason, full proofs are given throughout. To the best of my knowledge, the global results summarized Theorem 5.10 are new in this generality, in particular the description of sets mapped to a point stated in terms that work well in DEG1 $1_{\text {loc }}$ as illustrated by Corollary 5.12. Already in [3], essentially the same properties were obtained, but only for deformations whose boundary values are continuous and admit a homeomorphic extension, which among other things provides a much more straightforward way to control of $y(\bar{\Omega})$, whereas we cannot even assume that $y(\partial \Omega)$ is defined.

Proofs of partially related results in Nonlinear Elasticity, notably [3], [44], 33] and [34], the degree-theoretic parts of [17] and, more recently, 
[5], mix topological arguments with fine properties of Sobolev functions. Here, the presentation is purely topological, including a notion of strictly orientation preserving maps based on the degree. It expands and complements some results of [40], and it also reveals that some of the auxiliary results of [44] and related papers can in fact be derived using only information about the degree.

We start with general properties of orientation preserving maps, defined topologically in terms of the degree. As illustrated by [5], this is also useful in settings with low Sobolev regularity, suitable subsets of $W_{+}^{1, p}$ with $d-1<p<d$. Such settings as treated in [44, [33], [16] and [5] still allow degree theory, but only for selective "good" open subsets of $\Omega$. In our notation below, $\mathcal{O}$ could actually play that role. However, the more difficult issue in this scenario is that $y$ than can only be assumed to be continuous on boundaries of good sets, not everywhere. For global theory, using only balls as good sets (like in [44 or 33]) does not suffice, because they in general cannot separate connected components. In any case, in this article, we will not pursue this topic further.

\subsection{Strictly orientation preserving maps.}

Definition 5.1 (non-degenerate, (strictly) orientation preserving). Let $\Omega \subset \mathbb{R}^{d}$ be open, let $y: \Omega \rightarrow \mathbb{R}^{d}$ be continuous and let

$$
\mathcal{O}:=\{A \subset \Omega \mid A \text { is open and } A \subset \subset \Omega\} .
$$

We say that $y$ is non-degenerate if for each non-empty $U \in \mathcal{O}$, there exist $A \in \mathcal{O}$ with $A \subset U$ and $z \in \mathbb{R}^{d} \backslash y(\partial A)$ with $\operatorname{deg}(y ; A ; z) \neq 0$. We say that $y$ is orientation preserving if $\operatorname{deg}(y ; \cdot ; \cdot) \geq 0$, i.e., $\operatorname{deg}(y ; A ; z) \geq$ 0 for all $A \in \mathcal{O}$ and all $z \in \mathbb{R}^{d} \backslash y(\partial A)$. If $y$ is non-degenerate and orientation preserving then $y$ is called strictly orientation preserving.

For instance, any homeomorphism $y$ is non-degenerate and has constant degree in $\{ \pm 1\}$ on its image. Up to a reflection to achieve degree +1 if necessary, it is also strictly orientation preserving.

Remark 5.2. The same topological notion of orientation preserving maps is used in [5]. By itself, it is often too weak to be useful because it allows examples where the degree is simply not defined on the image of $y$ on all of $\Omega$ or large subsets. The attributes "non-degenerate" and "strictly orientation preserving" as defined here are not standard. The latter is similar but not equivalent to "locally sense-preserving" in the sense of, e.g., [40] (cf. Remark 5.9). "Non-degenerate" essentially expresses that $y$ is not allowed to compress open sets to sets with empty interior.

Remark 5.3. As we will see later, for $p \geq d$, deformations $y \in W_{+}^{1, p}$ are always strictly orientation preserving in the sense of Definition 5.1 . 
The case $p=d$ is the main reason we want $\mathcal{O}$ to be the family of open sets compactly contained in $\Omega$ instead of all open $A \subset \Omega$. As a consequence, it is not needed to have $y$ continuous up to the boundary.

For strictly orientation preserving maps in the sense of Definition 5.1 , both the local and the global degree are actually positive for any admissible value in the image of $y$ :

Lemma 5.4. Let $\Omega \subset \mathbb{R}^{d}$ be open, let $\mathcal{O}=\{A \subset \subset \Omega \mid A$ is open $\}$, let $y \in C\left(\Omega ; \mathbb{R}^{d}\right)$ be strictly orientation preserving in the sense of Definition 5.1, and let $z \in y(\Omega)$. Then for all connected components $C_{z}$ of $y^{-1}(\{z\})$ with $C_{z} \subset \subset \Omega$, there exists a sequence of sets $A_{n} \in \mathcal{O}$ such that for every $n \in \mathbb{N}, A_{n+1} \subset A_{n}$,

$$
\begin{aligned}
& C_{z} \subset A_{n}, \operatorname{Dist}\left(C_{z}, \partial A_{n}\right) \leq \frac{1}{n}, \\
& z \notin y\left(\partial A_{n}\right) \text { and } \operatorname{deg}\left(y ; A_{n} ; z\right) \geq 1 .
\end{aligned}
$$

If we also have that $\Omega$ is bounded and $y \in C\left(\bar{\Omega} ; \mathbb{R}^{d}\right)$, then

$$
\operatorname{deg}(y ; \Omega ; z) \geq 1 \text { for all } z \in y(\Omega) \backslash y(\partial \Omega) .
$$

Proof. Since $C_{z}$ is a compact subset of $\Omega$, it has a positive distance to $\partial \Omega$. Choose a sequence of open sets $U_{n} \in \mathcal{O}$ with

$$
C_{z} \subset U_{n} \subset \subset \Omega \text { and } \operatorname{Dist}\left(C_{z}, \partial U_{n}\right) \leq \frac{1}{n} .
$$

The set

$$
S_{n}:=\bar{U}_{n} \cap y^{-1}(\{z\})
$$

is compact. If $S_{n}=C_{z}$ then $y^{-1}(\{z\}) \cap \partial U_{n}=\emptyset$ and we take $A_{n}:=U_{n}$. Otherwise, $S_{n}$ is not connected and can be separated into two disjoint compact subsets $T_{n}$ and $R_{n}=S_{n} \backslash T_{n}$ such that

$$
C_{z} \subset T_{n} \text { and } S_{n} \cap \partial U_{n} \subset R_{n}
$$

(Whyburn Lemma, see [46, (9.3) in Chap. I, p. 12]). Since $T_{n}$ and $R_{n}$ have positive distance, there exists $A_{n} \in \mathcal{O}, A_{n} \subset U_{n}$ with $C_{z} \subset T_{n} \subset$ $A_{n}$ and $R_{n} \cap \partial A_{n}=\emptyset$. In all cases, we conclude that

$$
C_{z} \subset A_{n}, \operatorname{Dist}\left(C_{z}, \partial A_{n}\right) \leq \frac{1}{n} \text { and } z \notin y\left(\partial A_{n}\right) .
$$

W.l.o.g., we may also assume that $A_{n+1} \subset A_{n}$ for all $n$. Since $y$ is strictly orientation preserving, there exists

$$
x_{n} \in A_{n} \text { with } y\left(x_{n}\right) \notin \partial A_{n} \text { and } \operatorname{deg}\left(y ; A_{n} ; y\left(x_{n}\right)\right) \geq 1,
$$

the latter because $\operatorname{deg}\left(y ; A_{n} ; y\left(x_{n}\right)\right)>0$ and the degree is integervalued. Due to (5.4), we also have that $\operatorname{dist}\left(x_{n} ; C_{z}\right) \rightarrow 0$, and thus $y\left(x_{n}\right) \rightarrow z$ by the (locally uniform) continuity of $y$. Using the additivity and continuity of the degree together with $\operatorname{deg}(y ; \cdot ; \cdot) \geq 0(y$ is orientation preserving), (5.5) implies that for all $n$ and every $k \geq n$ 
large enough so that $y\left(x_{k}\right) \notin \partial A_{n}$ (which is possible since $y\left(x_{k}\right) \rightarrow z$ and $z$ has a positive distance to the compact set $\partial A_{n}$ for fixed $n$ ),

$$
\begin{array}{r}
1 \leq \operatorname{deg}\left(y ; A_{k} ; y\left(x_{k}\right)\right) \leq \operatorname{deg}\left(y ; A_{n} \backslash \bar{A}_{k} ; y\left(x_{k}\right)\right)+\operatorname{deg}\left(y ; A_{k} ; y\left(x_{k}\right)\right) \\
=\operatorname{deg}\left(y ; A_{n} ; y\left(x_{k}\right)\right) \underset{k \rightarrow \infty}{\longrightarrow} \operatorname{deg}\left(y ; A_{n} ; z\right) .
\end{array}
$$

We conclude that $\operatorname{deg}\left(y ; A_{n} ; z\right) \geq 1$ for all $n$. Combined with (5.4), this concludes the proof of (5.1).

Finally, if $y$ is continuous up to the boundary and $z \in y(\Omega) \backslash y(\partial \Omega)$, then $y^{-1}(\{z\}) \cap \Omega \neq \emptyset$ and any of its connected components $C_{z}$ is compactly contained in $\Omega$. Thus, $\operatorname{deg}(y ; \Omega ; z) \geq \operatorname{deg}\left(y ; A_{n} ; z\right) \geq 1$ by additivity of the degree.

Lemma 5.5 (on topological image and reduced domain).

Let $\Omega \subset \mathbb{R}^{d}$ be open, let $\mathcal{O}=\{A \subset \subset \Omega \mid A$ is open $\}$, let $y \in C\left(\Omega ; \mathbb{R}^{d}\right)$, let $A, A_{1}, A_{2} \in \mathcal{O}$ and let $U \subset \Omega$ be open. We define the open sets

$$
\begin{aligned}
& \operatorname{im}_{T}(y ; A):=\left\{z \in \mathbb{R}^{d} \backslash y(\partial A) \mid \operatorname{deg}(y ; A ; z) \neq 0\right\}, \\
& \operatorname{im}_{l o c}(y ; U):=\bigcup_{A \in \mathcal{O}, A \subset U} \operatorname{im}_{T}(y ; A)
\end{aligned}
$$

and the associated (topologically) reduced domain

$$
\mathcal{R}_{y}(U):=\{x \in U \mid \exists A \in \mathcal{O}: x \in A \subset U \text { and } y(x) \notin y(\partial A)\} .
$$

Then we have the following:

(i) $\operatorname{im}_{T}(y ; A) \subset \operatorname{int} y(A)$, the interior of $y(A)$.

(ii) If $y$ is orientation preserving then

$$
A_{1} \subset A_{2} \Longrightarrow \operatorname{im}_{T}\left(y ; A_{1}\right) \subset \operatorname{im}_{T}\left(y ; A_{2}\right) \cup y\left(\partial A_{2}\right) .
$$

(iii) If $y$ is strictly orientation preserving, then

$$
\begin{aligned}
& \operatorname{im}_{T}(y ; A)=y(A) \backslash y(\partial A), \\
& \operatorname{im}_{l o c}(y ; U) \subset y(U) \subset \overline{\operatorname{im}_{l o c}(y ; U)}, \\
& U \backslash \mathcal{R}_{y}(U) \text { has empty interior, } \\
& y\left(\mathcal{R}_{y}(U)\right)=\operatorname{im}_{l o c}(y ; U), \text { and } \\
& y\left(\bar{U} \backslash \mathcal{R}_{y}(U)\right) \subset y(\partial U) .
\end{aligned}
$$

If we have in addition that $\Omega$ is bounded and $y \in C\left(\bar{\Omega} ; \mathbb{R}^{d}\right)$, then all of the above also holds for $A=A_{2}=U=\Omega$.

Remark 5.6. In context of Nonlinear Elasticity, the concept of the topological image $\operatorname{im}_{T}$ (without the name) was introduced in [44]. We use it here without artificially adding the image of the boundary, which makes it an open set (due to continuity of the degree). This has the disadvantage that in general, full monotonicity with respect to the domain cannot be expected for even in case of strictly orientation preserving maps, only what follows from Lemma 5.5 (ii) and (iii). The "localized" topological image $\operatorname{im}_{\text {loc }}(y ; U)$ fixes this issue by collecting all points in local topological images. A variant of it was also used in [39]. 
As shown in Theorem 5.10 below, for strictly orientation preserving maps with global degree $\leq 1, \operatorname{im}_{\text {loc }}(y ; U)$ coincides with $\operatorname{im}_{T}(y ; U)$ as long as $y(\partial U)$ has empty interior. In addition, $\operatorname{im}_{\text {loc }}(y ; U)$ is a natural substitute of $y(U) \backslash y(\partial U)$. Unlike the latter, it remains meaningful with $U=\Omega$ even if $y$ cannot be continuously extended to $\partial \Omega$, and it possibly contains more information in cases where $\left.y\right|_{\partial U}$ exhibits wild behavior like Peano curves.

Remark 5.7. As Lemma 5.5 and Theorem 5.10 show, the topologically reduced domain $\mathcal{R}_{y}$ introduced in the lemma is always naturally associated to $\operatorname{im}_{\text {loc }}(y ; \cdot)$. By its definition and the continuity of $y, \mathcal{R}_{y}(U)$ is open. Moreover, since we can always isolate connected components with an arbitrarily close surrounding neighborhood unless they touch the boundary (cf. Lemma 5.4),

$$
\mathcal{R}_{y}(U)=\Lambda:=\bigcup_{z \in \mathbb{R}^{d}, C_{z} \in \operatorname{ICC}(z, U)} C_{z},
$$

where $\operatorname{ICC}(z, U)$ denotes the family of all "inner" connected components of $y^{-1}(\{z\}) \cap U$, i.e.,

$$
\operatorname{ICC}(z, U):=\left\{\begin{array}{l|l}
C_{z} & \begin{array}{l}
C_{z} \text { is a connected component of } y^{-1}(\{z\}) \cap U \\
\text { with } C_{z} \subset \subset U
\end{array}
\end{array}\right\}
$$

This also entails that for every $z \in y(\partial U)$ and every component $C_{z}$ of $y^{-1}(\{z\}) \cap U$ with $C_{z} \not \subset \mathcal{R}_{y}(U)$, we have that $C_{z} \cap \mathcal{R}_{y}(U)=\emptyset$ and $\bar{C}_{z} \cap \partial U \neq \emptyset$. Interestingly, it is not obvious that $\Lambda$ is open as defined.

Remark 5.8. Another basic property of $\mathcal{R}_{y}$ to keep in mind when working in DEG1 $1_{\text {loc }}$ is that for every covering $\left(\Omega_{m}\right)_{m \in \mathbb{N}}$ of $\Omega$ with open sets $\Omega_{m} \subset \Omega_{m+1} \subset \subset \Omega$,

$$
\mathcal{R}_{y}\left(\Omega_{m}\right) \subset \mathcal{R}_{y}\left(\Omega_{m+1}\right) \quad \text { and } \quad \mathcal{R}_{y}(\Omega)=\bigcup_{m \in \mathbb{N}} \mathcal{R}_{y}\left(\Omega_{m}\right),
$$

a straightforward consequence of the definition of $\mathcal{R}_{y}$. Similarly,

$$
\operatorname{im}_{\mathrm{loc}}\left(y ; \Omega_{m}\right) \subset \operatorname{im}_{\mathrm{loc}}\left(y ; \Omega_{m+1}\right) \quad \text { and } \operatorname{im}_{\mathrm{loc}}(y ; \Omega)=\bigcup_{m \in \mathbb{N}} \operatorname{im}_{\mathrm{loc}}\left(y ; \Omega_{m}\right) \text {. }
$$

Remark 5.9 (Connections to the results of Titus and Young [40]). The set $\mathcal{R}_{y}(\Omega)$ is related to concepts appearing in [40]. If we knew that

$$
y\left(\Omega \backslash \mathcal{R}_{y}(\Omega)\right) \text { is closed with empty interior }
$$

for a strictly orientation preserving $y$, then $y$ would belong to the class of functions called $\Omega$ in [40] with the corresponding set $C_{f}:=\Omega \backslash \mathcal{R}_{y}(\Omega)$ for $f=y$ (and our domain $\Omega$ ). However, unless $\mathcal{R}_{y}(\Omega)=\Omega$, (5.9) is extremely unnatural even if $y$ is continuous up to the boundary and $y(\partial \Omega)$ has empty interior; as a matter of fact, it even implies that $\mathcal{R}_{y}(\Omega)=\Omega$ in our setting. In essence, the issue reflects that [40] was written for manifolds without boundary. Nevertheless, the results of [40] do apply to $\left.y\right|_{\mathcal{R}_{y}(\Omega)}\left(\right.$ with $f=y, N=\mathcal{R}_{y}(\Omega), M=\mathbb{R}^{d}$ and $\left.C_{f}=\emptyset\right)$. 
As is, 40] effectively cannot provide any information on $\Omega \backslash \mathcal{R}_{y}(\Omega)$ without additional assumptions. On such assumption is that $y$ is light, i.e, $y^{-1}(\{z\})$ is totally disconnected for all $z$, but this again implies that $\Omega=\mathcal{R}_{y}(\Omega)$.

By contrast, Theorem 5.10 and Corollary 5.12 discuss $y$ on $\Omega \backslash \mathcal{R}_{y}(\Omega)$, and we already know that $\Omega \backslash \mathcal{R}_{y}(\Omega)$ has empty interior by Lemma 5.5 (iii) (and Remark 5.8), essentially a consequence of our stronger notion of strictly orientation preserving maps. Unlike the latter, the notion of sense preserving used in [40] never holds at points $x \in \Omega \backslash \mathcal{R}_{y}(\Omega)$ because it requires the connected component $C_{x}$ of $x$ in $y^{-1}(\{y(x)\})$ to be compact in $\Omega$ (in other words, $C_{x}$ must not touch $\partial \Omega$ ). On $\mathcal{R}_{y}(\Omega)$, it is weaker than "strictly orientation preserving", a consequence of Lemma 5.4.

Proof of Lemma 5.5. Notice that $\operatorname{im}_{T}(y ; A)$ is open by the continuity of the degree. Consequently, $\operatorname{im}_{\mathrm{loc}}(y ; U)$ is open as the union of open sets.

(i) This is clear since $\operatorname{im}_{T}(y ; A)$ is open and $\operatorname{im}_{T}(y ; A) \subset y(A)$ by the solvability property of the degree.

(ii) Let $z \in \operatorname{im}_{T}\left(y ; A_{1}\right)$. We may assume that $z \notin y\left(\partial A_{2}\right)$, because otherwise the assertion is obvious. By definition of $\operatorname{im}_{T}\left(y ; A_{1}\right), z \notin$ $y\left(\partial A_{1}\right)$ and $\operatorname{deg}\left(y ; A_{1} ; z\right) \neq 0$. Since $y$ is orientation preserving, we even have that $\operatorname{deg}\left(y ; A_{1} ; z\right)>0$, an we also know that $\operatorname{deg}\left(y ; A_{2} \backslash \bar{A}_{1} ; z\right) \geq 0$. By additivity of the degree, this implies that

$$
\operatorname{deg}\left(y ; A_{2} ; z\right)=\operatorname{deg}\left(y ; A_{2} \backslash \bar{A}_{1} ; z_{0}\right)+\operatorname{deg}\left(y ; A_{1} ; z\right)>0 .
$$

Consequently, $z \in \operatorname{im}_{T}\left(y ; A_{2}\right)$.

(iii) $\operatorname{im}_{\boldsymbol{T}}(\boldsymbol{y} ; \boldsymbol{A})=\boldsymbol{y}(\boldsymbol{A}) \backslash \boldsymbol{y}(\boldsymbol{\partial A})$ ": "С" is clear by definition, and " $\supset$ " is a consequence of Lemma 5.4.

"im loc $(\boldsymbol{y} ; \boldsymbol{U}) \subset \boldsymbol{y}(\boldsymbol{U})$ ": This follows from (i).

"y(U) $\subset \overline{\operatorname{im}_{\text {loc }}(\boldsymbol{y} ; \boldsymbol{U})}$ ": Let $z \in y(U)$, and choose a point $x \in U$ with $y(x)=z$. Since $U$ is open, there exists positive radii $r(k) \rightarrow 0$ such that $B_{r(k)}(x) \subset \subset U$ for each $k$. As $y$ is strictly orientation preserving, there exist open $A_{k} \subset B_{r(k)}(x)$ and $x_{k} \in A_{k}$ with $y\left(x_{k}\right) \notin y\left(\partial A_{k}\right)$ and $\operatorname{deg}\left(z ; A_{k} ; y\left(x_{k}\right)\right) \neq 0$. By construction of the sets $A_{k}$, we have that $x_{k} \rightarrow x$ as $k \rightarrow \infty$. Consequently, $z=y(x)=\lim _{k} y\left(x_{k}\right) \in \overline{\operatorname{im}_{\mathrm{loc}}(y ; U)}$. "U $\backslash \mathcal{R}_{\boldsymbol{y}}(\boldsymbol{U})$ has empty interior": Let $V \subset U \backslash \mathcal{R}_{y}(U)$ be open. By definition of $\mathcal{R}_{y}(U)$, we infer that $y(x) \in y(\partial A)$ for all $x \in V$ and all $A \in \mathcal{O}$ with $x \in A \subset U$. But since $y$ is strictly orientation preserving, only empty $V$ can satisfy this.

" $\boldsymbol{y}\left(\boldsymbol{\mathcal { R }}_{\boldsymbol{y}}(\boldsymbol{U})\right)=\operatorname{im}_{\mathrm{loc}}(\boldsymbol{y} ; \boldsymbol{U})$ ": For every $x \in \mathcal{R}(y ; U)$ choose an admissible associated $A \in \mathcal{O}$ from its definition, and let $C_{x}$ denote the connected component of $y^{-1}(y(x))$ containing $x$. Since $y(x) \notin y(\partial A)$, $C_{x}$ is a compact subset of $A$. By Lemma 5.4, there exists $A_{1} \in \mathcal{O}$ 
with $C_{x} \subset A_{1} \subset A, y(x) \notin y\left(\partial A_{1}\right)$ and $\operatorname{deg}\left(y ; A_{1} ; y(x)\right) \geq 1$. Hence, $y(x) \in \operatorname{im}_{T}\left(y ; A_{1}\right) \subset \operatorname{im}_{\text {loc }}(y ; U)$. Conversely, if $z \in \operatorname{im}_{\text {loc }}(y ; U)$, then $z \in \operatorname{im}_{T}(y ; A)$ for an $A \in \mathcal{O}, A \subset U$ with $z \notin y(\partial A)$. In addition, $\operatorname{deg}(y ; A ; z) \neq 0$, and consequently, there exists $x \in A$ with $y(x)=z$. We infer that $x \in \mathcal{R}(y ; U)$ and therefore $z=y(x) \in y(\mathcal{R}(y ; U))$.

"y $\left(\overline{\boldsymbol{U}} \backslash \boldsymbol{\mathcal { R }}_{\boldsymbol{y}}(\boldsymbol{U})\right) \subset \boldsymbol{y}(\boldsymbol{\partial U})$ ": It suffices to show that $\bar{U} \backslash y^{-1}(y(\partial U)) \subset$ $\bar{U} \backslash \mathcal{R}_{y}(U)$. Consider an arbitrary $x \in \bar{U}$ with $y(x) \notin y(\partial U)$. Then the connected component $C_{x}$ of $x$ in $\bar{U} \cap y^{-1}(y(x))$ has positive distance to $\partial U$. By Lemma 5.4, there exists $A \in \mathcal{O}$ with $C_{x} \subset A \subset U$ and $y(x) \notin y(\partial A)$. Hence, $x \in \mathcal{R}_{y}(U)$, and since $\mathcal{R}_{y}(U)$ is open, we even get that $x \notin \bar{U} \backslash \mathcal{R}_{y}(U)$.

The case $y \in C\left(\bar{\Omega} ; \mathbb{R}^{d}\right)$ : In this case, the proofs of (i) and (iii) still work for $A=U=\Omega$. The same is true concerning (ii), as long as we can still show that $\operatorname{deg}\left(y ; \Omega \backslash \bar{A}_{1} ; z\right) \geq 0$ for $z \notin y(\partial \Omega)$. Here, notice that $A:=\Omega \backslash \bar{A}_{1} \notin \mathcal{O}$ is not admissible in our definition of orientation preserving maps. Instead, choose a sequence $\Omega_{m} \in \mathcal{O}$ such that $\bar{\Omega}_{m} \subset \Omega$ and Dist $\left(\Omega_{m}, \partial \Omega\right) \rightarrow 0$ as $m \rightarrow \infty$. Since $y$ is uniformly continuous, $z \notin y\left(\bar{\Omega} \backslash \Omega_{m}\right)$ for $m$ large enough. By additivity of the degree, this implies that $\operatorname{deg}\left(y ; \Omega \backslash \bar{A}_{1} ; z\right)=\operatorname{deg}\left(y ; \Omega_{m} \backslash \bar{A}_{1} ; z\right) \geq 0$. The inequality holds because $y$ is orientation preserving and $\Omega_{m} \backslash \bar{A}_{1} \in \mathcal{O}$.

5.2. Strictly orientation preserving maps of degree one. The following theorem is of particular interest from the point of view of Nonlinear Elasticity, because it provides a major step towards the invertibility of deformations. It summarizes the topological properties of strictly orientation preserving maps with degree $\leq 1$. Among other things, it asserts that they are essentially monotone in the topological sense (see (ii) below). The only possible case where the preimage of a value can have several connected components occurs when all of these are contracted by the deformation to boundary points where the deformed configuration is in self-contact (cf. (iii)). These are exactly the pieces missing in the (topologically) reduced domain $\mathcal{R}_{y}$ defined and studied in Lemma 5.5, cf. Remark 5.7.

Theorem 5.10. Let $\Omega \subset \mathbb{R}^{d}$ be open, let $\mathcal{O}=\{A \subset \subset \Omega \mid A$ is open $\}$, let $y \in C\left(\Omega ; \mathbb{R}^{d}\right)$ be strictly orientation preserving in the sense of Definition [5.1, let $U \in \mathcal{O}$ and let $\operatorname{im}_{T}, \operatorname{im}_{l o c}$ and $\mathcal{R}_{y}$ be defined as in Lemma 5.5. In addition, assume that $\operatorname{deg}(y ; U ; \cdot) \leq 1$. Then we have the following:

(i) Let $A \in \mathcal{O}$ with $A \subset U$. Then $\operatorname{deg}(y ; A ; \cdot) \geq 1$ on $y(A) \backslash y(\partial A)$, and $\operatorname{deg}(y ; A ; \cdot) \leq 1$ on $y(A) \backslash[y(\partial A) \cup y(\partial U)]$. If $y(\partial U)$ has empty interior, then $\operatorname{deg}(y ; A ; \cdot)=1$ on $y(A) \backslash y(\partial A)$.

(ii) For all $z \in \operatorname{im}_{l o c}(y ; U) \backslash y(\partial U)$,

$$
\mathcal{R}_{y}(U) \cap y^{-1}(\{z\}) \text { is connected. }
$$


(iii) If $y(\partial U)$ has empty interior, then

$$
\operatorname{im}_{l o c}(y ; U) \cap y(\partial U)=\emptyset .
$$

The latter implies that

$$
\begin{aligned}
& \mathcal{R}_{y}(U)=U \backslash y^{-1}(y(\partial U)), \\
& \operatorname{im}_{l o c}(y ; U)=y(U) \backslash y(\partial U)=\operatorname{im}_{T}(y ; U) \text { and } \\
& y(\partial U)=\partial\left(\operatorname{im}_{l o c}(y ; U)\right) .
\end{aligned}
$$

If we have in addition that $\Omega$ is bounded and $y \in C\left(\bar{\Omega} ; \mathbb{R}^{d}\right)$, then we may also use $U=\Omega$.

If we can find one $U$ such that $y(\partial U)$ has empty interior, then smaller sets with a reasonable boundary inherit this property - a topological analogue of Lusin's property $(\mathrm{N})$ :

Corollary 5.11. Under the assumptions of Theorem 5.10, suppose in addition that int $y(\partial U)=\emptyset$. Then

$$
\operatorname{int} K=\emptyset \quad \Longrightarrow \quad \operatorname{int} y(K)=\emptyset, \quad \text { for all compact } K \subset \bar{U} \text {. }
$$

In particular, for any open and bounded $\tilde{U} \subset U$ with int $\partial \tilde{U}=\emptyset$, all assertions in Theorem 5.10 (i) and (iii) hold for $\tilde{U}$ instead of $U$.

If $\Omega$ is bounded and $y \in C\left(\bar{\Omega} ; \mathbb{R}^{d}\right)$, then $U=\Omega$ is also admissible.

The next corollary summarizes our results for the case $y \in D E G 1_{\text {loc }}$. We now also assume that $\Omega$ is bounded to avoid complications that would appear otherwise.

Corollary 5.12. Let $\Omega \subset \mathbb{R}^{d}$ be open and bounded, let $y \in C\left(\Omega ; \mathbb{R}^{d}\right)$ be strictly orientation preserving in the sense of Definition 5.1. let $\mathcal{O}=\{A \subset \subset \Omega \mid A$ is open $\}$ and let $\operatorname{im}_{T}, \operatorname{im}_{l o c}$ and $\mathcal{R}_{y}$ be defined as in Lemma 5.5. In addition, assume that $y \in \mathrm{DEG}_{\text {loc }}$ with respect to a regular inner covering $\left(\Omega_{m}\right)_{m \in \mathbb{N}}$ of $\Omega$, and that $y\left(\partial \Omega_{m}\right)$ has empty interior for all $m$. Then the following holds:

(i) For all $A \in \mathcal{O}, \operatorname{deg}(y ; A ; \cdot)=1$ on $y(A) \backslash y(\partial A)$.

(ii) For all $z \in y\left(\mathcal{R}_{y}(\Omega)\right), \mathcal{R}_{y}(\Omega) \cap y^{-1}(\{z\})$ is connected and compactly contained in $\mathcal{R}_{y}(\Omega)$.

(iii) For all $z \in y(\Omega) \backslash y\left(\mathcal{R}_{y}(\Omega)\right)$ and every connected component $C_{z}$ of $y^{-1}(\{z\}), \bar{C}_{z} \cap \partial \Omega \neq \emptyset$.

(iv) $\operatorname{im}_{l o c}(y ; \Omega)=y\left(\mathcal{R}_{y}(\Omega)\right)$ and $\Omega \backslash \mathcal{R}_{y}(\Omega)$ has empty interior.

If we have in addition that $y \in C\left(\bar{\Omega} ; \mathbb{R}^{d}\right)$, then $\operatorname{im}_{\text {loc }}(y ; \Omega)=y(\Omega) \backslash$ $y(\partial \Omega)$ and $\operatorname{deg}(y ; \Omega ; \cdot)=1$ on $\operatorname{im}_{l o c}(y ; \Omega)$.

Remark 5.13 (Interface to the analytical setting). Corollary 5.12 is meant to be applied to functions $y \in \mathcal{Y}$ in the effective admissible set $\mathcal{Y} \subset\{E<\infty\}$ of a variational minimization problem for a functional $E$, for instance a nonlinear elastic energy set up in a Sobolev space 
(e.g., $\mathcal{Y} \subset W_{+}^{1, p}$ for $p \geq d$ ). The following ingredients are needed to make this work:

(a) An embedding of $\mathcal{Y}$ into $C\left(\Omega ; \mathbb{R}^{d}\right)$ (cf. Remark 6.4);

(b) a global invertibility constraint or a boundary condition which implies $\mathcal{Y} \subset \mathrm{DEG}_{\text {loc }}$ (cf. Remark 2.19);

(c) $y$ is strictly orientation preserving for $y \in \mathcal{Y}$ (cf. Lemma 6.7);

(d) a way to prove that $y\left(\partial \Omega_{m}\right)$ has empty interior for each $m$. Since $\mathcal{L}^{d}\left(\partial \Omega_{m}\right)=0$ by our definition of a regular inner covering, Lusin's condition (N) is sufficient (cf. Remark 6.4).

If $y \in C\left(\bar{\Omega} ; \mathbb{R}^{d}\right)$, then for $(\mathrm{d})$, by Corollary 5.11 , it also suffices to have that $y(\partial \Omega)$ has empty interior.

Remark 5.14. Without the global invertibility constraint $\operatorname{deg}(y ; U ; \cdot) \leq$ 1, the assertions of Theorem 5.10 can fail to hold even locally. One example (from [3]) is the strongly orientation preserving "angle-doubling" map $y_{0}: \Omega:=B_{1}(0) \rightarrow B_{1}(0)$ for $d=2$, defined in complex polar coordinates by $y_{0}\left(r e^{i \varphi}\right)=r e^{2 i \varphi}$. It satisfies $\operatorname{deg}\left(y_{0} ; A ; z\right)=2$ for all $z$ close to $0=y_{0}(0)$ and all open $A \subset \Omega$ with $0 \in A$. Therefore, (i) does not hold. Moreover, for each $z \in B_{1}(0) \backslash\{0\}, y_{0}^{-1}(\{z\})=\left\{x_{1}, x_{2}\right\} \subset \Omega$ contains exactly two (antipodal) points, and thus is not connected as asserted in (ii). In addition, we can always choose $U \in \mathcal{O}$ with smooth boundary such that $x_{1} \in U$ and $x_{2} \in \partial U$. This violates (iii), as $y\left(x_{1}\right) \in \operatorname{im}_{\text {loc }}\left(y_{0} ; U\right), y\left(x_{2}\right) \in y_{0}(\partial U)$ and $y\left(x_{1}\right)=y\left(x_{2}\right)=z$.

Proof of Theorem 5.10. (i) Let $z \in y(A) \backslash y(\partial A)$. By Lemma 5.4, $\operatorname{deg}(y ; \tilde{A} ; z) \geq 1$ for a suitable $\tilde{A} \in \mathcal{O}$ with $\tilde{A} \subset A$ and $z \notin y(\partial \tilde{A})$. Since $\operatorname{deg}(y ; \cdot ; \cdot) \geq 0$, we infer that

$$
1 \leq \operatorname{deg}(y ; \tilde{A} ; z) \leq \operatorname{deg}(y ; A ; z)
$$

by the additivity of the degree. If, in addition, $z \notin y(\partial U)$, we analogously get that

$$
\operatorname{deg}(y ; A ; z) \leq \operatorname{deg}(y ; U ; z) \leq 1 .
$$

Now suppose that $y(\partial U)$ has empty interior, and let $S$ be an arbitrary connected component of $y(A) \backslash y(\partial A)$. In view of (5.11), it suffices to show that $\operatorname{deg}(y ; A ; \cdot) \leq 1$ on $S . A \operatorname{Asg}(y ; A ; \cdot) \geq 1$ on $y(A) \backslash y(\partial A)$ by (5.11) and $\operatorname{deg}(y ; A ; \cdot)=0$ on $\mathbb{R}^{d} \backslash y(\bar{A}), y(A) \backslash y(\partial A)$ is open by continuity of the degree. Hence, $S$ is open, too, and $S \backslash y(\partial U) \neq \emptyset$. Consequently, (5.12) implies that $\operatorname{deg}\left(y ; A ; z_{0}\right) \leq 1$ for a $z_{0} \in S$. Since $\operatorname{deg}(y ; A ; \cdot)$ is constant on $S$, again by continuity of the degree, we conclude that $\operatorname{deg}(y ; A ; z) \leq 1$ for all $z \in S$.

(ii) The proof is indirect. Let $z \in \operatorname{im}_{\text {loc }}(y ; U) \backslash y(\partial U)$ and suppose that $\mathcal{R}_{y}(U) \cap y^{-1}(\{z\})$ is not connected. We therefore have at least two connected components, say, $C_{z}^{1}$ and $C_{z}^{2}$, both of which are compact subsets of $U$. By Lemma 5.4, there exist disjoint sets $A^{1}, A^{2} \in$ 
$\mathcal{O}$ with $A^{1} \cup A^{2} \subset U, C_{z}^{j} \subset A^{j}, z \notin \partial A^{j}$ and $\operatorname{deg}\left(y ; A^{j} ; z\right) \geq 1$. Since $z \notin y\left(\partial U \cup \partial A^{1} \cup \partial A^{2}\right)$, by additivity of the degree and the fact that $\operatorname{deg}(y ; \cdot ; \cdot) \geq 0$ ( $y$ is orientation preserving), we obtain that $\operatorname{deg}\left(y ; \mathcal{R}_{y}(U) ; z\right) \geq \operatorname{deg}\left(y ; A^{1} ; z\right)+\operatorname{deg}\left(y ; A^{2} ; z\right) \geq 2$. This contradicts our assumption on the degree.

(iii) $\operatorname{im}_{\text {loc }}(\boldsymbol{y} ; \boldsymbol{U}) \cap \boldsymbol{y}(\boldsymbol{\partial U})=\emptyset "$ : The proof is indirect. Let $x_{0} \in \partial U$ and suppose that $z_{0}:=y\left(x_{0}\right) \in \operatorname{im}_{\text {loc }}(y ; U)$. By definition of $\operatorname{im}_{\text {loc }}(y ; U)$, there exists $A \in \mathcal{O}, A \subset U$ with $z_{0} \notin y(\partial A)$ and $\operatorname{deg}\left(y ; A ; z_{0}\right) \neq 0$. In particular, $y(x)=z_{0}$ for an $x \in A$ and $x \in \mathcal{R}_{y}(U)$. Let $C$ denote the connected component of $y^{-1}\left(z_{0}\right)$ containing $x$. Since $C$ is compact and $C \subset A, C \subset \mathcal{R}_{y}(U)$ by definition of the latter set. In particular, $C$ has positive distance to $\mathbb{R}^{d} \backslash \mathcal{R}_{y}(U) \supset \partial U$. By Lemma 5.4, we can find $A_{0} \in \mathcal{O}$ with $A_{0} \subset A$ such that

$$
\bar{A}_{0} \subset \mathcal{R}_{y}(U), z_{0} \notin y\left(\partial A_{0}\right) \text { and } \operatorname{deg}\left(y ; A_{0} ; z_{0}\right) \geq 1 .
$$

Now choose a sequence $\left(\tilde{x}_{k}\right) \subset U$ with $\tilde{x}_{k} \rightarrow x_{0} \in \partial U$ as $k \rightarrow \infty$. Let

$$
r(k):=\frac{1}{2} \operatorname{dist}\left(\tilde{x}_{k} ; \partial U\right) \leq \frac{1}{2}\left|\tilde{x}_{k}-x_{0}\right| \rightarrow 0 .
$$

Since $y$ is strictly orientation preserving, there exist sets $A_{k} \in \mathcal{O}$ such that for a $\tilde{z}_{k} \in \mathbb{R}^{d}$,

$$
A_{k} \subset B_{r(k)}\left(\tilde{x}_{k}\right), \quad \tilde{z}_{k} \notin y\left(\partial A_{k}\right) \text { and } \operatorname{deg}\left(y ; A_{k} ; \tilde{z}_{k}\right) \geq 1 .
$$

By continuity of the degree and the fact that $y\left(\partial A_{k}\right)$ is compact, (5.14) even holds for all $z_{k} \in V_{k}$ in place of $\tilde{z}_{k}$, in an open neighborhood $V_{k}$ of $\tilde{z}_{k}$. Shrinking $V_{k}$ if necessary, we can also make sure that $V_{k} \cap y\left(\partial A_{k}\right)=$ $\emptyset$. Since, by assumption, $y(\partial U)$ has empty interior while $V_{k}$ is open, there exists $z_{k} \in V_{k}$ with $z_{k} \notin y(\partial U)$. In addition, as $\operatorname{deg}\left(y ; A_{k} ; z_{k}\right) \neq 0$, $A_{k} \cap y^{-1}\left(z_{k}\right) \neq \emptyset$. Consequently, there exists at least one connected component $C_{k}$ of $y^{-1}\left(z_{k}\right)$ in $A_{k}$, and by definition of $\mathcal{R}_{y}(U)$, we also have that $C_{k} \subset \mathcal{R}_{y}(U)$.

On the other hand, $\sup _{x \in A_{k}}\left|x-x_{0}\right| \leq \frac{3}{2}\left|x_{0}-\tilde{x}_{k}\right| \rightarrow 0$ by construction. Since $\bar{A}_{0} \subset U$ while $x_{0} \in \partial U$, this implies that $A_{k} \cap A_{0}=\emptyset$ for all $k$ large enough, and we also infer that $z_{k}=y\left(x_{k}\right) \rightarrow y\left(x_{0}\right)=z_{0}$ by continuity of $y$, for arbitrary $x_{k} \in C_{k} \subset A_{k} \cap y^{-1}\left(\left\{z_{k}\right\}\right.$. Arguing as before, we obtain that (5.13) also holds for $z_{k}$ instead of $z_{0}$, for all sufficiently large $k$. As a consequence, besides $C_{k} \subset A_{k} \cap \mathcal{R}_{y}(U), y^{-1}\left(\left\{z_{k}\right\}\right)$ has a second connected component $\hat{C}_{k}$, now contained in $A_{0} \cap \mathcal{R}_{y}(U)$. Since we made sure that $z_{k} \notin y(\partial U)$, this contradicts (ii).

$" \boldsymbol{\mathcal { R }}_{\boldsymbol{y}}(\boldsymbol{U}) \supset \boldsymbol{U} \backslash \boldsymbol{y}^{-1}(\boldsymbol{y}(\boldsymbol{\partial U}))$ ": Let $z \in y(U) \backslash y(\partial U)$, and let $C_{z}$ denote an arbitrary connected component of $y^{-1}(\{z\})$. Since $z \notin y(\partial U)$, $C_{z}$ is compact and has a positive distance to $\partial U$. As a consequence of Lemma 5.4, $C_{z} \subset \mathcal{R}(y ; U)$.

"R $(\boldsymbol{y} ; \boldsymbol{U}) \subset \boldsymbol{U} \backslash \boldsymbol{y}^{-1}(\boldsymbol{y}(\boldsymbol{\partial U}))$ ": By definition, $\mathcal{R}(y ; U) \subset U$, and we already know that $\operatorname{im}_{\text {loc }}(y ; U) \cap y(\partial U)=\emptyset$ and $y(\mathcal{R}(y ; U))=$ 
$\operatorname{im}_{\text {loc }}(y ; U)$, the latter by Lemma 5.5 (iii). Hence,

$$
\mathcal{R}(y ; U) \subset y^{-1}\left(\operatorname{im}_{\mathrm{loc}}(y ; U)\right) \subset y^{-1}(y(U) \backslash y(\partial U))=U \backslash y^{-1}(y(\partial U)) .
$$

$" \operatorname{im}_{\mathrm{loc}}(\boldsymbol{y} ; \boldsymbol{U})=\boldsymbol{y}(\boldsymbol{U}) \backslash \boldsymbol{y}(\boldsymbol{\partial U}) ":$ Recall that $\operatorname{im}_{\mathrm{loc}}(y ; U)=y\left(\mathcal{R}_{y}(U)\right)$ by Lemma 5.5 (iii). Hence, $\operatorname{im}_{\text {loc }}(y ; U) \subset y(U)$, and we have just proved that $\operatorname{im}_{\text {loc }}(y ; U) \cap y(\partial U)=\emptyset$. We also already know that $U \backslash$ $y^{-1}(y(\partial U)) \subset \mathcal{R}(y ; U)$, which implies that $y(U) \backslash y(\partial U) \subset y\left(\mathcal{R}_{y}(U)\right)=$ $\operatorname{im}_{\mathrm{loc}}(y ; U)$.

$" \operatorname{im}_{\mathrm{loc}}(\boldsymbol{y} ; \boldsymbol{U})=\operatorname{im}_{\boldsymbol{T}}(\boldsymbol{y} ; \boldsymbol{U})$ ": By the definition of $\operatorname{im}_{T}, \operatorname{im}_{T}(y ; U) \subset$ $y(U) \backslash y(\partial U)$. We also have that $\operatorname{im}_{\text {loc }}(y ; U) \subset \operatorname{im}_{T}(y ; U) \cup y(\partial U)$, by the definition of $\operatorname{im}_{\text {loc }}(y ; U)$ and Lemma 5.5 (ii) with $A_{2}=U$. As $y(U) \backslash y(\partial U)=\operatorname{im}_{\text {loc }}(y ; U)$ due to the previous step, we infer both "つ" and "C".

$" \boldsymbol{y}(\boldsymbol{\partial U})=\boldsymbol{\partial}\left(\operatorname{im}_{\mathrm{loc}}(\boldsymbol{y} ; \boldsymbol{U})\right)$ ": Since $y(U) \backslash y(\partial U)=\operatorname{im}_{\text {loc }}(y ; U)$ is open and $y(\bar{U})$ is closed, it suffices to show that $y(\partial U) \subset \overline{\operatorname{im}_{\text {loc }}(y ; U)}$. By continuity of $y$, this follows from Lemma 5.5 (iii).

The case $\boldsymbol{U}=\Omega$ for $\boldsymbol{y} \in \boldsymbol{C}\left(\bar{\Omega} ; \mathbb{R}^{\boldsymbol{d}}\right)$ : For such a $y$, all notions used above are also defined for $U=\Omega$, and the proof works without changes. In particular, Lemma 5.5 can be applied with $A_{2}=U=\Omega$.

Proof of Corollary 5.11. The proof is indirect. Let $K \subset \bar{U}$ be compact with int $K=\emptyset$ and suppose there exists a non-empty open set $V \subset y(K)$. In particular, $V \backslash y(\partial U) \neq \emptyset$.

Since $y$ is continuous, there exists $x_{0} \in K \cap U$ and an open neighborhood $A_{0}$ of $x_{0}$ such that $y\left(A_{0}\right) \subset V$. Moreover, int $y\left(A_{0}\right) \neq \emptyset$ by the solvability and stability properties of the degree, exploiting that $y$ is strictly orientation preserving. Choose a non-empty open set

$$
A \subset \tilde{A}_{0}:=U \cap y^{-1}\left(\operatorname{int} y\left(A_{0}\right)\right) \neq \emptyset \text { such that } A \cap K=\emptyset .
$$

This is possible because $\tilde{A}_{0}$ is open and $K$ is closed with empty interior. Now let $x \in A$ and $z:=y(x)$. Since $y\left(A_{0}\right) \subset V \subset y(K), y^{-1}(\{z\})$ also contains a point $x_{2} \in K$. Let $C_{1}$ and $C_{2}$ be the connected components of $y^{-1}(\{z\}) \cap U$ with $x \in C_{1}$ and $x_{2} \in C_{2}$, respectively. By Theorem 5.10 (ii) and (iii), we have one of the following two possibilities:

(a) $\bar{C}_{1} \subset U$ and $C_{1}=C_{2}$, or

(b) $\bar{C}_{1} \cap \partial U \neq \emptyset$ and $\bar{C}_{2} \cap \partial U \neq \emptyset$.

In both cases, $\partial A \cap C_{1} \neq \emptyset$, because $C_{1}$ is connected, contains $x \in A$ and has to reach another point outside of $A$. With the same argument, we even get that $\partial \tilde{A} \cap C_{1} \neq \emptyset$ for all open $\tilde{A} \subset A$ with $x \in \tilde{A}$. In other words, $y(x) \in y(\partial \tilde{A})$ for all $x \in \tilde{A} \subset A$. But for strictly orientation preserving and therefore non-degenerate $y$, this is impossible.

Proof of Corollary 5.12. Recall that by Remark 5.8,

$$
\mathcal{R}_{y}(\Omega)=\bigcup_{m} \mathcal{R}_{y}\left(\Omega_{m}\right) \text { and } \operatorname{im}_{\mathrm{loc}}(y ; \Omega)=\bigcup_{m} \operatorname{im}_{\mathrm{loc}}\left(y ; \Omega_{m}\right) .
$$


The assertions (i)-(iii) now follow from Theorem 5.10 and Corollary 5.11. As to (ii) and (iii) also see Remark 5.7. For (iv), we use (5.15) and Lemma 5.5 (iii) with $U=\Omega_{m}$ for each $m$.

We conclude the section with a result that will be useful to exploit extra regularity of deformations with finite distortion while avoiding additional assumptions near the boundary.

Lemma 5.15. Let $\Omega \subset \mathbb{R}^{d}$ be open, and assume that $y \in C\left(\Omega ; \mathbb{R}^{d}\right)$ is strictly orientation preserving. If $U \subset \Omega$ is open, the restriction

$$
\hat{y}:=\left.y\right|_{\Lambda}: \Lambda \rightarrow \mathbb{R}^{d}, \quad \text { with } \Lambda:=\mathcal{R}_{y}(U),
$$

is strictly orientation preserving on $\Lambda$. If, in addition, $y$ is continuous on $\bar{U}$, then

$$
\operatorname{deg}(y ; U ; z)=\operatorname{deg}(\hat{y} ; \Lambda ; z) \quad \text { for all } z \notin y(\partial U) \supset y(\partial \Lambda) .
$$

Proof. The way we defined strictly orientation preserving maps, any restriction like $\hat{y}$ just means fewer sets $A$ to test with and thus trivially inherits this property. Now suppose that $y$ is continuous on $\bar{U}$. If $z \notin y(\partial U)$

$$
\operatorname{deg}(y ; U ; z)=\operatorname{deg}(y ; \emptyset ; z)+\operatorname{deg}(y ; \Lambda ; z)=\operatorname{deg}(y ; \Lambda ; z)
$$

by additivity of the degree. Here, notice that $y(\partial \Lambda) \subset y(\bar{U} \backslash \Lambda) \subset$ $y(\partial U)$, the latter by Lemma 5.5 (iii).

\section{Global invertibility in $W^{1, p}$}

Throughout this section, we will impose the global invertibility constraint $y \in \mathrm{DEG}_{\mathrm{loc}}$, or $y \in \mathrm{DEG} 1$ if $y$ is continuous up to the boundary, for all admissible deformations $y \in W_{+}^{1, p}$. Recall that by Remark 2.19, this assumption can always be replaced by any of the other invertibility constraints of Section 2, including the Ciarlet-Nečas condition $y \in \mathrm{CNC}$ and approximate invertibility on the boundary $y \in \mathrm{AIB}$ or $y \in \mathrm{AIB}_{\text {loc }}$. In the latter two cases, we have to assume in addition that $\mathbb{R}^{d} \backslash \partial \Omega$ has only two connected components to be able to apply Theorem 4.2.

For numerical purposes, $\mathrm{AIB}$ and $\mathrm{AI}(\bar{\Omega})$ are more accessible than the other constraints (cf. [24]).

6.1. Ball's global invertibility revisited. The invertibility results obtained in [3] all rely on the assumption that on $\partial \Omega$, the deformation $y$ (denoted $u$ in [3]) coincides with a continuous $u_{0}: \bar{\Omega} \rightarrow y(\bar{\Omega})$ which is injective on $\Omega$. By the results of Section 5 , it actually suffices to assume that $y \in$ DEG1 instead. This leads to the following generalization of [3, Theorem 1]: 
Theorem 6.1. Let $\Omega \subset \mathbb{R}^{d}$ be a bounded Lipschitz domain. Moreover, let $p>d$, let $y \in C\left(\bar{\Omega} ; \mathbb{R}^{d}\right) \cap W_{+}^{1, p}\left(\Omega ; \mathbb{R}^{d}\right) \cap$ DEG1. Then we have the following:

(i) $y(\bar{\Omega})=\overline{y(\Omega) \backslash y(\partial \Omega)}$ and $y(\partial \Omega)=\partial(y(\Omega) \backslash y(\partial \Omega))$.

(ii) For every measurable $A \subset \Omega$ and every measurable function $f$,

$$
\int_{A} f(y(x)) \operatorname{det} \nabla y(x) d x=\int_{y(A)} f(z) d z,
$$

as long as at least one of the two integrals exists.

(iii) For almost every $z \in y(\bar{\Omega}), y^{-1}(\{z\})$ consists of a single point.

(iv) If $z \in y(\Omega) \backslash y(\partial \Omega)$, then $y^{-1}(\{z\}) \subset \bar{\Omega}$ is a connected set contained in $\Omega$.

(v) $y^{-1}(y(\partial \Omega)) \cap \Omega$ has empty interior, and if $z \in y(\partial \Omega)$, then each of the connected components of $y^{-1}(\{z\}) \cap \Omega$ touches $\partial \Omega$.

A proof is given at the end of the subsection.

Remark 6.2. Since $\Omega$ is a Lipschitz domain, $y \in W^{1, p}\left(\Omega ; \mathbb{R}^{d}\right)$ always has a continuous representative in $C\left(\bar{\Omega} ; \mathbb{R}^{d}\right)$ by embedding, and $y$ can always be extended to a function in $W^{1, p}$ on a bigger domain.

Remark 6.3. With $f \equiv 1$ and $A=\Omega$, Theorem 6.1 (ii) implies the Ciarlet-Nečas condition, cf. Definition 2.14.

Remark 6.4 (The case $p=d$ ). Theorem 6.1 can be extended to the case $p=d$ with minor modifications, see Theorem 6.6. In this case, continuity of $y$ up to the boundary would be an unnatural extra assumption even for smooth domains. However, even if we only have that $y \in W_{+}^{1, d}\left(\Omega ; \mathbb{R}^{d}\right)$, we can still follow the proof of Theorem 6.1 in subdomains compactly contained in $\Omega$, exploiting Remark 5.8 and the following facts: Inside $\Omega$, deformations $y \in W_{+}^{1, d}$ automatically have a continuous representative [43] (cf. [11, Theorem 5.14], [44]) and satisfy Lusin's condition (N) [27, Corollary 3.13] (cf. [11, Theorem 5.32], or [17. Theorem 4.5]). Even an explicit modulus of continuity can be obtained at any given positive distance from the boundary [38] (cf. [11, Corollary 5.19]). In particular, any such $y$ can still be approximated by smooth functions, simultaneously in $W^{1, d}$ and locally uniformly. (The approximants do not necessarily have positive determinant, though.) For suitable extensions of the change of variables formulas (6.2) and (6.3) used below, see [11, Theorem 5.35 and Theorem 5.34] (e.g.).

By Theorem 4.2, Theorem6.1 immediately implies the following variant in the class of approximately invertible maps on the boundary in the sense of Definition 2.2 .

Corollary 6.5. Let $\Omega \subset \mathbb{R}^{d}$ be a bounded bounded Lipschitz domain such that $\mathbb{R}^{d} \backslash \partial \Omega$ has exactly two connected components. If $p>d$ and $y \in W_{+}^{1, p}\left(\Omega ; \mathbb{R}^{d}\right) \cap \mathrm{AIB}$, then the assertions (i)-(v) of Theorem 6.1 hold. 
In view of Remark 6.4, we can also easily obtain an extension of Theorem 6.1 for the case $p=d$ and without requiring $\Omega$ to be Lipschitz, with an analogous proof. The only difference is a weaker description of $y(\Omega)$, taking into account that we can no longer apply Theorem 5.10 or Lemma 5.5 with $U=\Omega$, only Corollary 5.12 which is obtained by approximating $\Omega$ from inside (see also Remark 5.8 and Remark 5.13). In $(\mathrm{v})$, the set $y(\Omega) \backslash \operatorname{im}_{\text {loc }}(y ; \Omega)$ now plays the role of $y(\Omega) \cap y(\partial \Omega)$ which is no longer defined.

Theorem 6.6. Let $\Omega \subset \mathbb{R}^{d}$ be open, let $p \geq d$ and suppose that $y \in$ $W_{+}^{1, p}\left(\Omega ; \mathbb{R}^{d}\right) \cap \mathrm{DEG} 1_{l o c}$, the latter with respect to a regular inner covering $\left(\Omega_{m}\right)_{m \in \mathbb{N}}$ of $\Omega$ (see Definition [2.9). With $\mathrm{im}_{T}$ and $\mathrm{im}_{l o c}$ defined as in Lemma 5.5, we then have the following:

(i) $\bigcup_{m \in \mathbb{N}} \operatorname{im}_{T}\left(y ; \Omega_{m}\right)=\operatorname{im}_{l o c}(y ; \Omega) \subset y(\Omega) \subset \overline{\operatorname{im}_{l o c}(y ; \Omega)}$.

(ii) For every measurable $A \subset \Omega$ and every measurable function $f$,

$$
\int_{A} f(y(x)) \operatorname{det} \nabla y(x) d x=\int_{y(A)} f(z) d z
$$

as long as at least one of the two integrals exists.

(iii) For almost every $z \in y(\Omega), y^{-1}(\{z\})$ consists of a single point.

(iv) If $z \in \operatorname{im}_{l o c}(y ; \Omega)$, then $y^{-1}(\{z\})$ is a compact connected set contained in $\Omega$.

(v) $\Omega \cap y^{-1}\left(y(\Omega) \backslash \operatorname{im}_{l o c}(y ; \Omega)\right)$ has empty interior, and if $z \in y(\Omega) \backslash$ $\operatorname{im}_{l o c}(y ; \Omega)$, then each of the connected components of $y^{-1}(\{z\}) \cap$ $\Omega$ touches $\partial \Omega$.

The following lemma links the analytical and topological notions of strictly orientation preserving maps.

Lemma 6.7. Let $\Omega \subset \mathbb{R}^{d}$ be open and bounded, let $p \geq d$ and let $y \in W_{+, \text {loc }}^{1, p}\left(\Omega ; \mathbb{R}^{d}\right)$. Then $y$ is strictly orientation preserving in the sense of Definition 5.1 .

Proof. Let $A \subset \subset \Omega$ be open. As remarked in [3], if $z \notin y(\partial A)$ and $V_{0}$ denotes the connected component of $\mathbb{R}^{d} \backslash y(\partial A)$ containing $z$, then the degree can be represented as

$$
\operatorname{deg}(y ; A ; z)=\int_{A} h(y(x)) \operatorname{det} \nabla y(x) d x,
$$

for any continuous $h: \mathbb{R}^{d} \rightarrow[0, \infty)$ compactly supported in $V_{0}$ and with $\int_{V_{0}} h(z) d z=1$. Notice that (6.1) is actually a special case of (6.2) below which uses that the degree is locally constant. It thus also extends to the case $p=d$, cf. Remark 6.4. As an immediate consequence of (6.1),

$$
\operatorname{deg}(y ; A ; z) \geq 0 \text { for every } z \in \mathbb{R}^{d} \backslash y(\partial A) .
$$

Hence, $y$ is orientation preserving in the sense of of Definition 5.1. To prove that it is so strictly, first notice that we may assume w.l.o.g. that 
$\mathcal{L}^{N}(\partial A)=0$, moving to a slightly smaller but more regular set if necessary. For $z \in y(A) \backslash y(\partial A)$, we can always choose a function $h$ such that $h(z)>0$. By continuity of $y, h \circ y>0$ on a neigborhood of $U \cap y^{-1}(\{z\})$ which automatically has positive measure. Using (6.1) once more, we infer that

$$
\operatorname{deg}(y ; U ; z)>0 \text { for every } z \in y(A) \backslash y(\partial A) .
$$

In addition, $\mathcal{L}^{d}(A)>0$ implies that $\mathcal{L}^{d}(y(A))>0$ by the area formula (6.3) (see also Remark 6.4 for $p=d$ ), while Lusin's property $(\mathrm{N})$ and $\mathcal{L}^{N}(\partial A)=0$ imply that $\mathcal{L}^{d}(y(\partial A))=0$. As long as $A \neq \emptyset$, we know that $\mathcal{L}^{d}(A)>0$ since $A$ is open, and consequently, $y(A) \backslash y(\partial A) \neq \emptyset$. We conclude that $y$ is also strictly orientation preserving in the sense of of Definition 5.1.

Proof of Theorem 6.1. The topological assertions (i), (iv) and (v) follow from Corollary 5.12. Its assumptions hold as described in Remark 5.13. $y \in \mathrm{DEG}_{\text {loc }}$ by Remark 2.19 (c), and $y$ is strictly orientation preserving in the sense of Definition 5.1 by Lemma 6.7 below. Due to Lusin's condition (N) [26] and the fact that $\mathcal{L}^{d}\left(\partial \Omega_{m}\right)=0$, we also know that for all $m, y\left(\partial \Omega_{m}\right)$ has measure zero and thus empty interior. In addition, we exploit some change-of-variables formulas to show (ii) and (iii) as in [3]. Assertion (ii) is a consequence of a more general change-of-variables formula valid for $y \in W^{1, p}\left(\Omega ; \mathbb{R}^{d}\right), p>d, A \subset \subset \Omega$ open with $\mathcal{L}^{d}(\partial A)=0$ and $f \in L^{\infty}\left(\mathbb{R}^{d}\right)$ :

$$
\int_{A} f(y(x)) \operatorname{det} \nabla y(x) d x=\int_{y(A)} f(z) \operatorname{deg}(y ; A ; z) d z,
$$

see [10, Theorem 5.31] (e.g.). In our case, for every $z \in y(\Omega)$ and every open $A \subset \subset \Omega, \operatorname{deg}(y ; A ; \cdot)=1$ on $y(A) \backslash y(\partial A)$ by Theorem 5.10 (i), and $y(\partial A)$ is always a set of measure zero. Hence, (6.2) implies assertion (ii) for every open $A \subset \subset \Omega$ with $\mathcal{L}^{d}(\partial A)=0$ and $f \in L^{\infty}\left(\mathbb{R}^{d}\right)$. The general case follows with an approximation argument. With the help of the area formula [26],

$$
\int_{A}|\operatorname{det} \nabla y(x)| d x=\int_{y(A)} \# y^{-1}(\{z\}) d z
$$

where \# denotes the counting measure, (ii) with $f \equiv 1$ implies (iii).

6.2. Improved invertibility exploiting finite distortion. Any map $y$ in $W_{+}^{1, p}\left(\Omega ; \mathbb{R}^{d}\right)$, or, more generally, in $W_{+, \text {loc }}^{1, p}\left(\Omega ; \mathbb{R}^{d}\right)$ is automatically a map of (almost everywhere) finite distortion, with outer distortion

$$
K_{y}^{O}(x):=|\nabla y(x)|^{d}(\operatorname{det} \nabla y(x))^{-1} .
$$

The inner distortion of $y$ is defined as

$$
K_{y}^{I}(x):=\left|(\nabla y(x))^{-1}\right|^{d} \operatorname{det} \nabla y(x)=|\operatorname{cof} \nabla y(x)|^{d}(\operatorname{det} \nabla y(x))^{1-d} .
$$


Here, $\operatorname{cof} \nabla y$ denotes the cofactor matrix of $\nabla y$, cf. Remark 6.18. We always have that $\left(K_{y}^{O}\right)^{d-1} \geq c K_{y}^{I}$ with a constant $c=c(d)>0$, because $|F|^{d-1} \geq c|\operatorname{cof} F|$ for all $F \in \mathbb{R}^{d \times d}$.

The investigation of maps with finite inner or outer distortion was initiated by [3, Theorem 2] (for $p>d$ ) and strongly influenced by 44] (in particular for $p=d$ ). Their theory is now well developed [17].

If $y \in W_{+}^{1, d}\left(\Omega ; \mathbb{R}^{d}\right), K_{y}^{O} \in L_{\text {loc }}^{q(d-1)}$ with $q>1$ and $y$ is not constant, then it is open and discrete [42, i.e., $y$ maps open sets to open sets in $\mathbb{R}^{d}$ and for any $z \in \mathbb{R}^{d}, y^{-1}(\{z\})$ does not have accumulation points in $\Omega$. A slightly stronger version of the same result was obtained in [37, for $K_{y}^{O} \in L_{\mathrm{loc}}^{d-1}$ and $K_{y}^{I} \in L_{\mathrm{loc}}^{q}$ with $q>1$.

This can be combined with Theorem 5.10 to generalize [17, Theorem 3.27] and the result sketched in [17, Remark 7.6]:

Theorem 6.8. Let $\Omega \subset \mathbb{R}^{d}$ be open, let $p \geq d$ and let $y \in W_{+, \text {loc }}^{1, p}\left(\Omega ; \mathbb{R}^{d}\right) \cap$ $C\left(\Omega ; \mathbb{R}^{d}\right) \cap \mathrm{DEG}_{\text {loc }}$ such that for a $q>1$, either $K_{y}^{O} \in L_{\text {loc }}^{q(d-1)}(\Omega)$, or $K_{y}^{O} \in L_{l o c}^{d-1}(\Omega)$ and $K_{y}^{I} \in L_{l o c}^{q}(\Omega)$. Then

$$
y: \Omega \rightarrow y(\Omega) \text { is a homeomorhpism }
$$

with inverse $y^{-1} \in W_{l o c}^{1, d}\left(y(\Omega) ; \mathbb{R}^{d}\right)$. If $K_{y}^{O} \in L^{d-1}(\Omega)$, we also have that $\nabla y^{-1} \in L^{d}\left(y(\Omega) ; \mathbb{R}^{d \times d}\right)$.

If, in addition, $\Omega$ is bounded, $y \in C\left(\bar{\Omega} ; \mathbb{R}^{d}\right)$ and $y(\partial \Omega)$ has empty interior, for example if $y \in W_{+}^{1, p}\left(\Omega ; \mathbb{R}^{d}\right)$ with $p>d$ and $\Omega$ is a Lipschitz domain, then DEG1 loc can be replaced by DEG1 above.

Remark 6.9. As pointed out in [15, Section 3] for $d=3$ (and easily extended to any $d$ ), openness combined with invertibility almost everywhere implies invertibility everywhere. This is essentially equivalent to Theorem 6.8, although its proof is completely different. For comparison, recall that for $y$ in $W_{+}^{1, p}$ with $p \geq d$, injectivity a.e. is equivalent to the Ciarlet-Nečas condition $y \in \mathrm{CNC}$. We know that $\mathrm{CNC}=\mathrm{DEG}_{\text {loc }}$ by Remark 2.19.

Proof of Theorem $\mathbf{6 . 8}$. Let $\left(\Omega_{m}\right)_{m \in \mathbb{N}}$ be the regular inner covering of $\Omega$ from the definition of DEG1 $1_{\text {loc }}$. Recall that $y$ always has a continuous representative in $\Omega$ and satisfies Lusin's condition $(\mathrm{N})$ ([26]; see also Remark 6.4 if $p=d)$. As a first consequence, $y\left(\partial \Omega_{m}\right)$ has measure zero and thus empty interior for each $m$. By Lemma 6.7, $y$ is strictly orientation preserving in the sense of Definition 5.1. In particular, $y$ is not constant. Now let $z \in y(\Omega)$, whence $z \in y\left(\Omega_{m}\right)$ for big enough $m$. Since $y$ is discrete by either [42, Theorem 1] or [37, Theorem 1], all connected components of $y^{-1}(\{z\})$ consist of a single point. By Remark 5.7, this entails that $R_{y}(\Omega)=\Omega$, and by Theorem 5.10 (ii) (applied with $U=\Omega_{m}$ for arbitrary $m$; see also Remark 5.8), we infer that $y^{-1}(\{z\})$ is a singleton. Hence, $y: \Omega \rightarrow y(\Omega) \subset \mathbb{R}^{d}$ is bijective, 
and since it is also continuous, it is a homeomorphism by Theorem A.2. The Sobolev regularity of $y^{-1}$ was shown in [17, Theorem 5.9].

If we only control the inner distortion of $y \in W_{+}^{1, d}\left(\Omega ; \mathbb{R}^{d}\right)$, similar results can be obtained given that $y$ is quasi-light, i.e., $y^{-1}(\{z\})$ is a compact subset of $\Omega$ for all $z \in y(\Omega)$. Then, $K_{y}^{I} \in L^{1}$ implies that $y$ is either constant or open and discrete [36]. Notice that since $y$ is continuous, quasi-light just means that for $z \in y(\Omega)$, there are no connected components of $y^{-1}(\{z\})$ touching $\partial \Omega$. In other words, no continuum connected to the boundary is compressed to a point. This holds by construction if we replace $\Omega$ by the reduced domain $\mathcal{R}_{y}(\Omega)$ of Lemma 5.5 (see also Remark [5.7). As a result, we can generalize [17, Theorem 7.5] as follows:

Theorem 6.10. Let $\Omega \subset \mathbb{R}^{d}$ be open, let $p \geq d$, let $y \in W_{+, \text {loc }}^{1, p}\left(\Omega ; \mathbb{R}^{d}\right) \cap$ $C\left(\Omega ; \mathbb{R}^{d}\right) \cap \mathrm{DEG}_{\text {loc }}$ with $K_{y}^{I} \in L_{\text {loc }}^{1}(\Omega)$. Then

$$
y: \mathcal{R}_{y}(\Omega) \rightarrow \operatorname{im}_{l o c}(y ; \Omega)=y\left(\mathcal{R}_{y}(\Omega)\right) \text { is a homeomorhpism }
$$

with $y^{-1} \in W_{l o c}^{1, d}\left(\operatorname{im}_{l o c}(y ; \Omega) ; \mathbb{R}^{d}\right)$. If $K_{y}^{I} \in L^{1}(\Omega)$, we also get that $\nabla y^{-1} \in L^{d}\left(\operatorname{im}_{l o c}(y ; \Omega) ; \mathbb{R}^{d \times d}\right)$.

If, in addition, $\Omega$ is bounded, $y \in C\left(\bar{\Omega} ; \mathbb{R}^{d}\right)$ and $y(\partial \Omega)$ has empty interior, for example if $y \in W_{+}^{1, p}\left(\Omega ; \mathbb{R}^{d}\right)$ with $p>d$ and $\Omega$ is a Lipschitz domain, then DEG1 $1_{l o c}$ can be replaced by DEG1 above and we also know that $\operatorname{im}_{\text {loc }}(y ; \Omega)=y(\Omega) \backslash y(\partial \Omega)=\operatorname{im}_{T}(y ; \Omega)$ and $\mathcal{R}_{y}(\Omega)=$ $\Omega \backslash y^{-1}(y(\partial \Omega))$.

Remark 6.11. It is indeed possible that $\Omega \backslash \mathcal{R}_{y}(\Omega) \neq \emptyset[18]$.

Proof of Theorem 6.10. Recall that $\mathcal{R}_{y}(\Omega) \subset \Omega$ is open and $\left.y\right|_{\mathcal{R}_{y}(\Omega)}$ is quasi-light by definition of $\mathcal{R}_{y}(\Omega)$ (cf. Remark [5.7), that $\left.y\right|_{\mathcal{R}_{y}(\Omega)}$ is also strictly orientation preserving in the topological sense like $y$ (Lemma 5.15) and that $\operatorname{im}_{\text {loc }}(y ; \Omega)=y\left(\mathcal{R}_{y}(\Omega)\right)$ by Theorem 5.10 (ii) and Remark 5.8. In view of these facts, using [37, Theorem 1] on $\tilde{\Omega}:=\mathcal{R}_{y}(\Omega)$ instead of $[42$ on $\Omega$, we argue analogously to the proof of Theorem 6.8 and infer that $y: \mathcal{R}_{y}(\Omega) \rightarrow \operatorname{im}_{\text {loc }}(y ; \Omega)$ is a homeomorphism. For the Sobolev regularity of its inverse, first notice that formally, $K_{y}^{I}(x)=\left|\left(\nabla y^{-1}\right)(y(x))\right|^{d} \operatorname{det} \nabla y(x)$. By [17, Theorem 5.2], we rigorously obtain that $y^{-1} \in W_{\mathrm{loc}}^{1, d}\left(\operatorname{im}_{\mathrm{loc}}(y ; \Omega) ; \mathbb{R}^{d}\right)$, and $\left\|K_{y}^{I}\right\|_{L^{1}(\Omega)}=$ $\left\|\nabla y^{-1}\right\|_{L^{d}\left(\operatorname{im}_{\mathrm{loc}}(y ; \Omega) ; \mathbb{R}^{d \times d}\right)}$ by change of variables.

If $p>d, y \in W_{+}^{1, p}\left(\Omega ; \mathbb{R}^{d}\right)$ and $\Omega$ is Lipschitz, then $y$ satisfies Lusin's property $(\mathrm{N})$ and $\mathcal{L}^{d}(y(\partial \Omega))=0$. Lemma 5.5 (iii) and Theorem 5.10 (iii) with $A=U=\Omega$ provide the asserted properties of $\operatorname{im}_{\text {loc }}(y ; \Omega)$ and $\mathcal{R}_{y}(\Omega)$.

Remark 6.12 (Connection to light maps). Unlike the argument of [15], the proofs of Theorem 6.8 and Theorem 6.10 do not really use that $y$ 
is open (or discrete), they use that

$$
y^{-1}(\{z\}) \text { is totally disconnected for each } z \in \mathbb{R}^{d} .
$$

This is obviously weaker than discreteness. In our setting for strictly orientation preserving maps in DEG1 or DEG1 $1_{\text {loc }}$, it also implies openness: (6.4) implies that $y$ is a homeomorphism by the argument in the proof of Theorem 6.8. Maps satisfying (6.4) are called light. By the Titus-Young theorem [40, Theorem A], strictly orientation preserving, light maps are always local (but not necessarily global) homeomorphisms on a dense open subset of the domain. Connections to our topological results are explained in Remark 5.9.

Remark 6.13. For maps that are local homeomorphisms everywhere, also in a suitable neighborhood of each boundary point, invertibility on the boundary is known to imply global invertibility [45]. This is still true if there are at most finitely many exceptional points inside the domain (and none on the boundary) around which local injectivity does not hold [29, Theorem 2]. The result of [45] does not require any topologocial restriction on the domain like we do in Theorem 4.2 .

6.3. Existence of homeomorphic minimizers. In this section, we present an existence result to demonstrate a typical application of our results. No attempt is made to achieve maximally general assumptions, and many other variants would be possible, too. In particular, there are natural generalizations for $d=2$ and $d \geq 4$ instead of $d=3$. The special case of controlled outer distortion with deformations subject to the Ciarlet-Nečas condition, (ii) below with $\mathcal{G}=\mathrm{CNC}$, is essentially already covered by the results of [15, Section 3]. Combined with Remark 2.19, their approach can also be used for other global invertibility constraints.

The model in the following theorem describes a nonlinearly elastic solid with reference configuration $\Omega$, enclosed in a rigid box whose interior is given by $\Lambda$. Interpenetration of matter is prevented both locally and globally, and depending on the shapes of $\Omega$ and $\Lambda$ - possibly very rough sets - this can lead to quite interesting, strongly deformed optimal configurations including self-contact of the elastic material. All contact is friction-less, but on a large scale, effectively friction-like forces can still be caused if $\Omega$ and $\Lambda$ are rough on comparatively small scales.

Theorem 6.14. Let $p \geq d=3$, let $\Omega, \Lambda \subset \mathbb{R}^{d}$ be open and bounded and suppose we have a functional (the sum of elastic and potential energy)

$$
E(y):=\int_{\Omega}(W(\nabla y)+g \cdot y) d x, \quad E: \mathcal{Y} \rightarrow(-\infty,+\infty],
$$

where the class of admissible deformations $y$ is given by

$$
\mathcal{Y}:=W_{+}^{1, p}\left(\Omega ; \mathbb{R}^{3}\right) \cap \mathcal{G} \cap\{y \mid y(\Omega) \subset \bar{\Lambda}\} .
$$


Here, $\mathcal{G}$ is one of the sets in $\left\{\mathrm{DEG} 1_{l o c}, \mathrm{CNC}, \mathrm{INV}, \mathrm{AIB}_{l o c}, \mathrm{AI}_{\text {loc }}(\Omega)\right\}(a$ global constraint preventing self-interpenetration). If $\mathcal{G}=\mathrm{AIB}_{\text {loc }}$, we also require that $\mathbb{R}^{3} \backslash \partial \Omega$ has only two connected components. As to the integrand of $E$, we assume that $g \in L^{1}\left(\Omega ; \mathbb{R}^{3}\right)$ (an external body force, say, gravity) and the following properties of $W: \mathbb{R}^{3 \times 3} \rightarrow[0,+\infty]$ (the stored energy density of the elastic body):

$W$ is continuous on $G L_{+}(3):=\left\{F \in \mathbb{R}^{3 \times 3} \mid \operatorname{det} F>0\right\}$;

$W(F)=+\infty \quad$ if and only if $F \in \mathbb{R}^{3 \times 3} \backslash G L_{+}(3)$;

$W(F) \rightarrow+\infty$ as $\operatorname{det} F \rightarrow 0$;

$W(F) \geq|F|^{p} \quad$ for all $F \in G L_{+}(3)$;

$W$ is polyconvex (cf. Remark 6.18).

Then $E$ attains its minimum in $\mathcal{Y}$, and every minimizer $y^{*} \in \mathcal{Y}$ is a.e. injective in $\Omega$. More can be said if the energy controls the inner or the outer distortion:

(i) If we have in addition that for all $F \in G L_{+}(3)$,

$$
W(F) \geq c \frac{|\operatorname{cof} F|^{3}}{(\operatorname{det} F)^{2}},
$$

with a constant $c>0$, then every minimizer $y^{*} \in \mathcal{Y}$ is a homeomorphism on $\mathcal{R}_{y^{*}}(\Omega) \subset \Omega$, the reduced domain of Lemma 5.5, and $y^{*}\left(\mathcal{R}_{y^{*}}(\Omega)\right)$ is open in $\mathbb{R}^{d}$ and thus a subset of $\Lambda$.

(ii) If we even have that for all $F \in G L_{+}(3)$,

$$
W(F) \geq c\left(\frac{|F|^{6}}{(\operatorname{det} F)^{2}}+\frac{|\operatorname{cof} F|^{3 q}}{(\operatorname{det} F)^{2 q}}\right),
$$

with constants $q>1$ and $c>0$, then every minimizer $y^{*} \in \mathcal{Y}$ is a homeomorphism on $\Omega, y^{*}(\Omega)$ is open and $y^{*}(\Omega) \subset \Lambda$.

Remark 6.15. For example, with $r>0$ and $s \geq 1$,

$$
W_{1}(F):=|F|^{p}+\frac{1}{(\operatorname{det} F)^{r}}, W_{2}(F):=|F|^{p}+\frac{1}{(\operatorname{det} F)^{r}}+|\operatorname{cof} F|^{s}
$$

and

$$
W_{3}(F):=|F|^{p}+\frac{1}{(\operatorname{det} F)^{r}}+|\operatorname{cof} F|^{s}+\frac{|F|^{6}}{(\operatorname{det} F)^{2}}
$$

all satisfy (6.5) - 6.9), where we set $W_{i}(F):=+\infty$ if $\operatorname{det} F \leq 0$. They are also frame indifferent in the sense that $W_{i}(Q F)=W_{i}(F)$ for all $F \in G L_{+}(3)$ and all rotations $Q \in S O(3)$. Moreover, (6.10) holds

- in case $W=W_{1}$, if $p>6$ and $r \geq \frac{2 p}{p-6}$, and

- in case $W=W_{2}$ or $W=W_{3}$ for any $p \geq 3$, if $r>2$ and $s \geq \frac{3 r}{r-2}$,

by Young's inequality. For $W=W_{1}$ and $W=W_{3}$, strict inequalities yield (6.11). 
Remark 6.16. If $\Omega \backslash \mathcal{R}_{y^{*}}(\Omega) \neq \emptyset$, then by the results of Section 5 , it consists of connected sets $C \subset \Omega$ in such a way that for each $C$, $C$ touches $\partial \Omega$ and $y^{*}$ compresses $C$ to a point in $y^{*}(\Omega) \backslash y^{*}\left(\mathcal{R}_{y^{*}}(\Omega)\right)$ $\left(\subset y^{*}(\partial \Omega)\right.$, if $y^{*}$ happens to be continuous up to the boundary). We also know that $\Omega \backslash \mathcal{R}_{y^{*}}(\Omega)$ is small in the sense that it has empty interior; it even has measure zero, because $y^{*}$ is a.e. invertible (because any $y \in \mathcal{Y}$ automatically satisfies the Ciarlet-Nečas condition $y \in \mathrm{CNC}$, see Remark 2.19). For more information on the set $\mathcal{R}_{y}(\Omega)$ see Lemma 5.5 , Theorem 5.10 and the Remarks 5.7 and 5.8 .

Remark 6.17. The theorem is written mostly for the case $p=d$; if we assumed and exploited $p>d$ and a Lipschitz domain $\Omega$, the results would be slightly stronger and simpler to state, and we could also admit the constraints $\mathcal{G}=\operatorname{AIB}$ (if $\Omega$ has connected boundary), $\mathcal{G}=\operatorname{AI}(\bar{\Omega})$ or $\mathcal{G}=$ DEG1.

Remark 6.18. Polyconvexity as required in (6.9) means that there exists a function

$$
\begin{aligned}
& h: \mathbb{R}^{3 \times 3} \times \mathbb{R}^{3 \times 3} \times(0, \infty) \rightarrow \mathbb{R} \text { convex, such that } \\
& W(F)=h(F, \operatorname{cof} F, \operatorname{det} F) \text { for all } F \in G L_{+}(3),
\end{aligned}
$$

cf. [4]. Here, cof $F$ denotes the cofactor matrix, i.e., a matrix formed of the determinants of all $2 \times 2$-submatrices of $F$. Usually, they are ordered and given suitable signs so that $(\operatorname{cof} F)^{T} F=\operatorname{det} F$, but this is irrelevant for our purposes.

Proof of Theorem 6.14. The existence of minimizers is a standard application of the direct method. First observe that there always is $y \in \mathcal{Y}$ with $E(y)<\infty$, for instance $\hat{y}(x):=z_{0}+\lambda x$, where $z_{0} \in \Lambda$ is chosen arbitrarily but fixed and $\lambda>0$ is small enough so that $\hat{y}(\Omega) \subset \Lambda$, exploiting that dist $\left(z_{0} ; \partial \Lambda\right)>0$.

By the constraint $y(\Omega) \subset \bar{\Lambda}, \mathcal{Y}$ is bounded in $L^{\infty}$. Hence, the linear force term $y \mapsto \int_{\Omega} g \cdot y d x$ is well defined, and by dominated convergence, it is also sequentially continuous in $\mathcal{Y}$, first with respect to pointwise convergence almost everywhere and then also with respect to weak convergence in $W^{1, p}$.

Using (6.8) and the a-priori bound in $L^{\infty}$, it is not difficult to show the coercivity estimate

$$
E(y) \geq c_{1}\|y\|_{W^{1, p}}^{p}-c_{2} \quad \text { for } y \in W^{1, p}\left(\Omega ; \mathbb{R}^{d}\right) \text { with } y(\Omega) \subset \bar{\Lambda},
$$

where $c_{1}>0$ and $c_{2}$ are real constants. By arguments of [32] (or [4] if $p>d=3$ ),

$$
\begin{array}{r}
y \mapsto \int_{\Omega} W(\nabla y) d x \text { is weakly sequ. lower semicontinuous } \\
\text { on } W^{1, p}\left(\Omega ; \mathbb{R}^{d}\right) \cap\{E<\infty\} .
\end{array}
$$

Here, the essential ingredients for the proof of (6.13) are the weak continuity of $y \mapsto \operatorname{det} \nabla y, W_{+}^{1, p} \rightarrow L^{1}$ on compact subsets of $\Omega$, the 
weak continuity of $y \mapsto \operatorname{cof} \nabla y, W_{+}^{1, p} \rightarrow L^{p / 2}$, and the convexity of $h$ (the polyconvexity of $W$, cf. Remark 6.18).

As a consequence of (6.12), any sequence $\left(y_{k}\right) \subset \mathcal{Y}$ with $E\left(y_{k}\right) \rightarrow I:=$ $\inf _{y \in \mathcal{Y}} E(y)<\infty$ is bounded and has a weakly convergent subsequence in $W^{1, p}$, say, $y_{k} \rightarrow y^{*}$. Due to (6.13), $E\left(y^{*}\right) \leq \lim _{k} E\left(y_{k}\right)=I$. As we also have that $y_{k} \rightarrow y^{*}$ locally uniformly (by embedding if $p>d=3$, or by Remark 6.4 if $p=3$ ), we obtain that $y^{*}(\Omega) \subset \bar{\Lambda}$. In addition, $\operatorname{det} \nabla y^{*}>0$ a.e. by (6.6), because $E\left(y^{*}\right)<\infty$. Hence, $y^{*} \in W_{+}^{1, p}$. Finally, $y^{*} \in \mathcal{G}$, because $\mathcal{G} \cap W_{+}^{1, p}$ is weakly sequentially closed in all cases (see Section 2). Altogether, $y^{*} \in \mathcal{Y}$ is a minimizer.

The remaining assertions (and some additional properties) follow from Theorem 6.10 and Theorem 6.8, repectively. The assumption on $K_{y^{*}}^{I}$ or $K_{y^{*}}^{O}$ are obtained from (6.10) or (6.11), and by Remark 2.19, the theorems can be applied for all possible choices of $\mathcal{G}$.

\section{Appendix A. The Problem of HomeOmorphic EXTEnsion}

When working with injective continuous maps, it is good to keep in mind the following two well-known facts.

Lemma A.1. Let $X, Z$ be topoogical spaces and $y: X \rightarrow Z$ continuous and injective, and suppose that $X$ is compact. Then $y: X \rightarrow y(X)$ is a homeomorphism, where $y(X)$ is endowed with the trace topology of $Z$.

Proof. By continuity of $y, y(X)$ is also compact. Open sets in $X$ and $y(X)$, respectively, are thus exactly the complements of compact sets. Since $y: X \rightarrow y(X)$ is bijective and maps compact sets to compact sets, it therefore also maps open sets in $X$ to open sets in $y(X)$.

The statement above does not mean that $y$ maps opens sets in $X$ to open sets in $Z$, because $y(X)$ is usually not open in $Z$. In $\mathbb{R}^{d}$, more can be said:

Theorem A.2. Let $\Omega \subset \mathbb{R}^{d}$ be open, $\Omega \neq \emptyset$, and let $y: \Omega \rightarrow \mathbb{R}^{n}$ be injective and continuous. Then $n \geq d$. Moreover, $y(\Omega)$ is open in $\mathbb{R}^{n}$ if and only if $n=d$. For $n=d, y: \Omega \rightarrow y(\Omega)$ is a homeomorphism.

Proof. This is a combination of the openness (invariance of domain) and invariance of dimension theorems based on the topological degree, see [11, Thm. 3.30, Cor. 3.31 and Cor. 3.32] (e.g.). The last assertion is a consequence of the others which also hold for arbitrary open subsets of $\Omega$, thereby proving that $y$ maps open subsets of $\Omega$ to open sets in $\mathbb{R}^{n}=\mathbb{R}^{d}$.

A.1. Homeomorphic extension versus Schoenflies extension. We are here mainly interested in homeomorphic extension for functions given on the boundary of some domain in $\mathbb{R}^{d}$. In the most general form, this problem reads as follows: 
Problem A.3 (Homeomorphic extension problem in $\mathbb{R}^{d}$ ). Let $\Omega \subset \mathbb{R}^{d}$ a bounded domain, and suppose that $y: \partial \Omega \rightarrow \mathbb{R}^{d}$ is continuous and injective. Is there a homeomorphism $h: \bar{\Omega} \rightarrow h(\bar{\Omega}) \subset \mathbb{R}^{d}$ such that $h=y$ on $\partial \Omega$ ?

Additional assumptions on topological nature of $\Omega$ and $\partial \Omega$ are typically added, as it is well-known that simple counterexamples exist when $\Omega$ is topologically complicated. For instance, on an annulus, homeomorphic extension is impossible if the winding numbers of $y$ on the two boundary pieces are not the same (e.g., one clockwise and the other counterclockwise).

A close relative is the following question which is much more widely studied in the literature:

Problem A.4 (Schoenflies extension problem). Let $y: S^{d-1} \rightarrow S^{d}$ continuous and injective. Is there a homeomorphism $h: S^{d} \rightarrow S^{d}$ such that $h\left(S^{d-1}\right)=\Sigma^{d-1}:=y\left(S^{d-1}\right)$, where $S^{d-1} \subset S^{d}$ is interpreted as the equator of the $d$-dimensional sphere $S^{d} \subset \mathbb{R}^{d+1}$ ?

The Schoenflies extension problem imposes a restriction on the topological type of admissible domains (one of the half-spheres separated by the equator) - it must be a topological ball - which is also commonly used for the homeomorphic extension problem. Apart from that, the two problems are essentially equivalent:

Proposition A.5 (Schoenflies versus homeomorphic extension).

Suppose that $\Omega \subset \mathbb{R}^{d}$ is a bounded domain such that there exists a homeomorphism $\gamma: \bar{\Omega} \rightarrow \bar{H}$, where $H \subset S^{d} \subset \mathbb{R}^{d+1}$ is one of the two hemispheres of $S^{d}$ separated by the "equator" $S^{d-1}$. Moreover, let $y: S^{d-1} \rightarrow S^{d}$ continuous and injective and let $\delta: S^{d} \rightarrow \mathbb{R}^{d} \cup\{\infty\}$ be a homeomorphism with $\delta\left(y\left(S^{d-1}\right)\right) \subset \mathbb{R}^{d}$, where $\mathbb{R}^{d} \cup\{\infty\}$ denotes the one-point compactification of $\mathbb{R}^{d}$. Then we have the following for $\tilde{y}:=\delta \circ y \circ \gamma$ :

(i) If there exists Schoenflies extension $h: S^{d} \rightarrow S^{d}$ of y as in Problem A.4, then a homeomorphic extension $\tilde{h}$ of $\tilde{y}$ as in Problem A.3 exists, too.

(ii) Conversely, if no Schoenflies extension $h: S^{d} \rightarrow S^{d}$ of y exists, a homeomorphic extension also fails to exist for one of the following two maps:

(a) $\tilde{y}: \partial \Omega \rightarrow \mathbb{R}^{d}$, or

(b) $\hat{y}:=\tilde{y} \circ \iota: \partial \hat{\Omega} \rightarrow \mathbb{R}^{d}$, with $\hat{\Omega}$ denoting the bounded connected component of $\mathbb{R}^{d} \backslash \iota(\partial \Omega)$.

Here, $\iota: \mathbb{R}^{d} \cup\{\infty\} \rightarrow \mathbb{R}^{d} \cup\{\infty\}, \iota(x):=\left|x-x_{0}\right|^{-2}\left(x-x_{0}\right)$, is the inversion map with respect to a point $x_{0} \in \mathbb{R}^{d}$; for (ii), we choose an arbitrary but fixed $x_{0} \in \Omega$. 
Proof. (i) $\tilde{\varrho}:=h^{-1} \circ y: S^{d-1} \rightarrow S^{d-1}$ is a homeomorphism of the equator $S^{d-1}$ onto itself. It has an explicit "radial" homeomorphic extension $\varrho: S^{d} \rightarrow S^{d}$. Using cylindrical coordinates $(x, t) \in S^{d-1} \times$ $[-1,1]$, it is given by

$$
\varrho\left(\left(1-t^{2}\right)^{\frac{1}{2}} x, t\right):=\left(\left(1-t^{2}\right)^{\frac{1}{2}} \tilde{\varrho}(x), t\right) \in S^{d} \subset \mathbb{R}^{d} \times \mathbb{R} .
$$

Using the inversion map $\iota$ with respect to a point $x_{0}$ in the bounded connected component of $\tilde{y}(\partial \Omega) \subset \mathbb{R}^{d}$, we now define $\tilde{h}:=\delta \circ h \circ \varrho \circ \gamma$ or $\tilde{h}:=\iota \circ \delta \circ h \circ \varrho \circ \gamma$. One of the two options satisfies $\infty \notin \tilde{h}(\bar{\Omega})$, and for this choice, $\left.\tilde{h}\right|_{\bar{\Omega}}$ is a homeomorphic extension of $\tilde{y}$ in the sense of Problem A.3.

(ii) If a Schoenflies extension $h: S^{d} \rightarrow S^{d}$ of $y$ does not exists, then extension already fails in one of the two hemispheres of $S^{d}$ separated by $S^{d-1}$, either $\bar{H}=\gamma(\bar{\Omega})$ or $S^{d} \backslash H$. (Otherwise, the extensions to the hemispheres can be glued to a Schoenflies extension, after first matching their parametrization of $y\left(S^{d-1}\right)$ using the radial homeomorphic extension of the proof of (i).) Accordingly, for either $\tilde{y}$ or $\hat{y}=\tilde{y} \circ \iota$, where $\iota$ is taken with respect to an $x_{0} \in \Omega$, there exists no homeomorphism defined on the closure of $\Omega$ or $\hat{\Omega}$, respectively, who maps the boundary of its domain to $\tilde{y}(\partial \Omega)=\hat{y}(\partial \hat{\Omega})$. In particular, either $\tilde{y}$ or $\hat{y}$ has no homeomorphic extension to its domain in the sense of Problem A.3.

A.2. Known results and counterexamples. For $d=2$, the answer to Problem A.4 is affirmative, given by the classical Schoenflies Theorem. Extension theorems for more regular classes of invertible functions are also known in this case, for instance bi-Lipschitz extensions [41, 9]. Recently, an extension result (also) valid in the class of Sobolev homeomorphisms has been established in [20, Theorem 4 and Corollary 5]. This is based on $p$-harmonic extension and even smooth in $\Omega$.

For $d \geq 3$, the situation is significantly more complicated. In general, a Schoenflies extension can fail to exist, for instance in case of Alexander's horned sphere [1. However, the result can be recovered for $d \geq 3$ if the embedded sphere $\Sigma^{d-1}:=y\left(S^{d-1}\right)$ is locally flat:

Theorem A.6 (Generalized Schoenflies Theorem [7, Theorem 4]). Let $\Sigma^{d-1} \subset S^{d}$ be a homeomorphic embedding of $S^{d-1}$ which is locally flat in the following sense:

For each $x_{0} \in \Sigma^{d-1}$, there exists a neighborhood $V$ of $x_{0}$ in $S^{d}$ and a homeomorphism $\zeta: V \rightarrow \zeta(V) \subset S^{d}$ s.t. $\zeta\left(V \cap \Sigma^{d-1}\right) \subset S^{d-1}$,

where $S^{d-1}$ is interpreted as the equator of $S^{d}$. Then $\Sigma^{d-1}$ is flat, i.e., there is a homeomorphism $h: S^{d} \rightarrow S^{d}$ such that $h\left(\Sigma^{d-1}\right)=S^{d-1}$. 
There are also variants of the Generalized Schoenflies Theorem that require higher regularity of $\Sigma^{d-1}$ instead of assuming a locally flat embedding. In particular, this is possible for the piecewise affine (polyhedral) [2] or diffeomorphic [28] case. As pointed out in [25, Example $3.10(5)$ ] for $d=3$, bi-Lipschitz regularity is not enough.

Remark A.7 (Homeomorphic extension may fail for $d \geq 3$ ). In view of Proposition A.5, [25, Example 3.10 (5)] also entails that for $d=$ 3 , homeomorphic extension is in general impossible even if the given boundary homeomorphism $y: \partial \Omega \rightarrow \Sigma^{d-1}:=y(\partial \Omega)$ is bi-Lipschitz.

Remark A.8. The example of [25] is based on the Fox-Artin arc [12, Example 1.1], a bi-Lipschitz embedding of a compact interval into $\mathbb{R}^{3}$ whose image has a complement which is not simply connected. By thickening it, surrounding the original interval by a domain consisting of two thin cones back-to-back with tips at the two end points of the interval, the self-similar construction yields a bi-Lipschitz mapping of the domain boundary onto a surface in $\mathbb{R}^{3}$. This surface is a topological 2-sphere, and from the Fox-Artin arc, it inherits that the unbounded component of its complement is not simply connected. In particular, a Schoenflies extension (after identifying $\mathbb{R}^{3} \cup\{\infty\}$ with $S^{3}$ ) is impossible because its existence would imply that both halves of $S^{3}$ separated by the surface are topological 3-balls which are simply connected.

On the other hand, if we look for a solution of Problem A.4 when the embedding of the sphere is known to be locally flat with higher regularity given in the whole neighborhood of its image $\Sigma^{d-1}$, then this regularity sometimes can be carried over to a suitable extension. In particular, this is possible in the bi-Lipschitz case [25, Theorem 7.7], or for the second order bi-Sobolev homeomorphisms where both the function and its inverse are in $W^{2, p}$ with $1 \leq p<d[14$. As far as I know, there is no comparable result for bi-Sobolev homeomorphisms in $W^{1, p}$ (yet?), only the theory for maps with finite distortion [17] which is conceptually closer to regularity theory than to extension results. In the diffeomorphic category, extensions starting from locally flat embeddings face another obstacle in higher dimensions, the possible existence of exotic spheres, for example for $d=8$ (7-dimensional spheres) [30, Theorem 3.4].

\section{A.3. Homeomorphic extension for $C^{1}$ functions on Lipschitz}

domains. As shown in Proposition A.5, a solution to Problem A.4 can be used to build homeomorphic extensions of maps $\left.y\right|_{\partial \Omega}$, at least if $\Omega$ is homeomorphic to the closed unit ball. A more practical application in the same spirit is given below, using Theorem A.6 to obtain a homeomorphic extension of a $C^{1}$-deformation on a Lipschitz domain which is invertible on the boundary. Despite the similarity, it does 
not directly follow from the Schoenflies extension for a $C^{1}$ map outlined in [28, p.11], because we would first have to transform the given Lipschitz domain to the unit ball. This is possible, but the transformation is only bi-Lipschitz and we would lose the crucial $C^{1}$ regularity (cf. Remark A.7).

Theorem A.9. Suppose that $\Omega \subset \mathbb{R}^{d}$ is a Lipschitz domain such that $\bar{\Omega}$ is homeomorphic to the closed unit ball. Moreover, let $y \in C^{1}\left(\bar{\Omega} ; \mathbb{R}^{d}\right)$ such that $\left.y\right|_{\partial \Omega}$ is injective and $\operatorname{det} \nabla y \neq 0$ on $\partial \Omega$. Then $\left.y\right|_{\partial \Omega}$ admits a homeomorphic extension to $\bar{\Omega}$.

Proof. The proof is based on Theorem A.6 and Proposition A.5(i). To apply the theorem, we identify $S^{d}$ with the one-point compactification $\mathbb{R}^{d} \cup\{\infty\}$ of $\mathbb{R}^{d}$. In this sense, $\mathbb{R}^{d} \subset S^{d}$ (homeomorphically embedded), and $y$ maps $\bar{\Omega}$ to $\mathbb{R}^{d} \subset S^{d}$, and $\Sigma^{d-1}:=y(\partial \Omega)$ is a homeomorphic embedding of a topological $(d-1)$-dimensional sphere into $\mathbb{R}^{d} \subset S^{d}$. To see that this embedding is also locally flat in the sense of Theorem A.6. it suffices to define local bi-Lipschitz extensions of $y \in C^{1}\left(\bar{\Omega} ; \mathbb{R}^{d}\right)$ in a neighborhood of each boundary point $x_{0} \in \partial \Omega$. Here, notice that $\partial \Omega$ is locally the graph of a Lipschitz function. This implies that $\partial \Omega$ is locally flat, and we may therefore assume that the homeomorphism mapping $\Omega$ to the unit ball is defined on a whole neighborhood of $\bar{\Omega}$.

Since $\partial \Omega$ is Lipschitz, we can choose a cylidrical neigborhood of the form $C_{\varepsilon}\left(x_{0}\right):=D_{\varepsilon}\left(x_{0}\right)+(-\varepsilon, \varepsilon) \nu \subset \mathbb{R}^{d}$ with a unit vector $\nu=\nu\left(x_{0}\right) \in$ $\mathbb{R}^{d}$ and a $(d-1)$-dimensional disc $D_{\varepsilon}\left(x_{0}\right)$ of radius $\varepsilon$, centered at $x_{0}$ and perpendicular to $\nu$. For $\varepsilon>0$ small enough and an appropriate choice of $\nu, \partial \Omega \cap C_{\varepsilon}\left(x_{0}\right)$ can be represented as the graph of a Lipschitz function $g: D_{\varepsilon}\left(x_{0}\right) \rightarrow(-\varepsilon, \varepsilon)$, such that $\Omega \cap C_{\varepsilon}\left(x_{0}\right)=\left\{x^{\prime}+t \nu \mid t<g\left(x^{\prime}\right)\right\}$. We can now extend $\left.y\right|_{\partial \Omega}$ to a function $\hat{y}: C_{\varepsilon}\left(x_{0}\right) \rightarrow \mathbb{R}^{d}$ by setting

$$
\hat{y}\left(x^{\prime}+t \nu\right):=y\left(x^{\prime}+g\left(x^{\prime}\right) \nu\right)+\left(t-g\left(x^{\prime}\right)\right) D y\left(x_{0}\right) \nu .
$$

for $x^{\prime} \in D_{\varepsilon}\left(x_{0}\right)$ and $t \in(-\varepsilon, \varepsilon)$. Close to $x_{0}$, this extension divides $C_{\varepsilon}\left(x_{0}\right)$ into surfaces of the form $\partial \Omega+s \nu$ (parametrized by $x^{\prime}+$ $g\left(x^{\prime}\right) \in \partial \Omega$ and $\left.s=t-g\left(x^{\prime}\right)\right)$ and maps each such surface onto $y(\partial \Omega)+s D y\left(x_{0}\right) \nu$, a shifted copy of $y(\partial \Omega)$.

To see that $\hat{y}$ is bi-Lipschitz in a neighborhood of $x_{0}$, the key observation is the following: Just like $\nu$ and $\partial \Omega, D y\left(x_{0}\right) \nu$ and the surface $y(\partial \Omega)$ always form an angle bounded away from zero as long as we remain close enough to $x_{0}$, because $D y$ is continuous and $D y\left(x_{0}\right)$ is invertible. As an immediate consequence, $\partial \Omega \rightarrow \mathbb{R}^{d}, \sigma \mapsto y(\sigma)+s D y\left(x_{0}\right)$ is injective near $x_{0}$ for each $s$, and in a small enough neighborhood $V$ of $y\left(x_{0}\right)$ in $\mathbb{R}^{d}$, we also have that

$$
V \cap\left[y(\partial \Omega)+s D y\left(x_{0}\right) \nu\right] \cap\left[y(\partial \Omega)+s_{2} D y\left(x_{0}\right) \nu\right]=\emptyset \quad \text { for } s_{1} \neq s_{2} .
$$

Hence, $\hat{y}$ is injective near $x_{0}$. Further details are omitted. 
Theorem A.6 now gives us a Schoenfliess extension of $\Sigma^{d-1}:=y(\partial \Omega) \subset$ $\mathbb{R}^{d} \cup\{\infty\} \cong S^{d}$, and by Proposition A.5 (i), this implies the existence of a homeomorphic extension of $\left.y\right|_{\partial \Omega}$ to $\bar{\Omega}$.

\section{Appendix B. Counterexamples for domains in With holes}

The following examples illustrate that the assumption that $\mathbb{R}^{d} \backslash \partial \Omega$ has only two connected components cannot be dropped in Theorem 4.2. For simplicity, they are all constructed for $d=2$, but they have straightforward equivalents in higher dimensions, still using domains with holes. In particular, it does not really matter whether $\Omega$ is simply connected or not. In both examples, the explicit values asserted for the degree are always taken at a suitable regular value of $y$ with just one pre-image $x_{0} \in \Omega$, and are therefore given as the sign of det $\nabla y\left(x_{0}\right)$. Geometric intuition provides a good heuristic, observing whether or not the local deformation is orientation-preserving. If yes, the sign is positive, otherwise negative.

Example B.1. Take the annulus $\Omega:=B_{2}(0) \backslash \bar{B}_{1}(0) \subset \mathbb{R}^{2}$ and for $x \in \Omega$ consider $y \in W^{1, \infty}\left(\Omega ; \mathbb{R}^{2}\right)$,

$$
y(x):=2 \frac{|x|-1}{|x|} x+\frac{2-|x|}{|x|}(x+(3,0)) .
$$

As defined, $y$ keeps the outer boundary $\partial B_{2}(0)$ fixed while translating the inner boundary onto $y_{1}\left(\partial B_{1}(0)\right)=(3,0)+\partial B_{1}(0)$. In particular, $\left.y\right|_{\partial \Omega}$ is invertible, but it maps $\partial \Omega$ to two circles that lie outside of each other. Now, $\mathcal{B}=B_{2}(0) \cup\left[(3,0)+B_{1}(0)\right]$ and $\sigma$ changes sign; more precisely, $\operatorname{deg}(y ; \Omega ;(0,0))=1$ while $\operatorname{deg}(y ; \Omega ;(3,0))=-1$.

It is also not enough to have that $\partial \Omega$ is connected:

Example B.2. Take a fixed unit vector $e \subset \mathbb{R}^{2}$ and truncated open cones of the form

$$
\hat{V}(\alpha, r):=O(r, \alpha) \cup\left\{x \in \mathbb{R}^{2}|x \cdot e>(1-\alpha)| x|,| x \mid<r\right\}, \alpha, r>0,
$$

where $O(r, \alpha) \subset \mathbb{R}^{s}$ denotes the unique open ball which touches the surface of the unbounded cone tangentially at $|x|=r$. Consequently, $V_{\alpha, r}$ has a boundary of class $C^{1}$ everywhere except at its tip in the origin. We create a domain by removing a smaller cone from a bigger one sharing the same tip:

$$
\Omega:=V_{2} \backslash \bar{V}_{1}, \quad \text { where } V_{s}:=\hat{V}\left(\frac{s}{3}, s\right) .
$$

As a first step, we now consider a map $y \in W^{1, \infty}\left(\Omega ; \mathbb{R}^{2}\right)$ which keeps the outer part of the boundary fixed while flipping the inner part outside, with affine interpolation on suitable rays in between. For its explicit definition, the flip is realized by the reflection $R$ across $\{x \cdot e=0\}$, $R x:=x-2(x \cdot e) e$, and we use the (nonlinear) projections $Q(x)$ and 
$P(x)$ onto the inner and the outer boundary, respectively, along lines perpendicular to the inner boundary $\partial V_{1}$ : For all $x \in \Omega \backslash\{0\}$,

$$
Q(x) \in \partial V_{1}, P(x) \in \partial V_{2} \text { and } P(x)-Q(x) \perp \partial V_{1} \text { at } Q(x) .
$$

Notice that $Q, P: \Omega \rightarrow \mathbb{R}^{2}$ are Lipschitz and thus in $W^{1, \infty}$ (even $C^{1}$ away from $x=0$ ), and both converge to the origin as $|x| \rightarrow 0, x \in \Omega$. We now can define

$$
\begin{array}{r}
y(x):=\frac{|x-Q(x)|}{|P(x)-Q(x)|} P(x)+\frac{|P(x)-x|}{|P(x)-Q(x)|} R[Q(x)] \\
=\left(1+\frac{|P(x)-x|}{|P(x)-Q(x)|}\right)(R[Q(x)]-P(x)) .
\end{array}
$$

The latter representation shows that $y$ is Lipschitz also at the origin.

This construction does not yet contradict Theorem 4.2, because as a matter of fact, $\operatorname{deg}(y ; \Omega ; \cdot)=1$ on both components of $\mathcal{B}\left(\mathbb{R}^{d} \backslash y(\partial \Omega)\right)=$ $V_{2} \cup\left(-V_{1}\right)$. In any case, with a second deformation that squeezes the line orthogonal to $e$ in the image to the origin and simultaneously reflects the half-space $\{x \cdot e<0\}$ containing $-V_{1}$ across the line in direction $e$, we can make the degree change sign at the value $-e \in-V_{1}$ while keeping it fixed at $e \in V_{2}$. More precisely, for

$$
\hat{y}:=F \circ y \quad \text { with } F(z):=(z \cdot e) e+(z \cdot e)\left(z \cdot e^{\perp}\right) e^{\perp},
$$

we have that $e,-e \in \mathcal{B}\left(\mathbb{R}^{d} \backslash \hat{y}(\partial \Omega)\right)$. Moreover, $F$ keeps the line in direction $e$ including those two points fixed, and they are regular values for both $F$ and $y$. Hence, $\operatorname{deg}(\hat{y} ; \Omega ; e)=+\operatorname{deg}(y ; \Omega ; e)=1$ and $\operatorname{deg}(\hat{y} ; \Omega ;-e)=-\operatorname{deg}(y ; \Omega ;-e)=-1$, because $\operatorname{det} \nabla F(-e)<0<$ $\operatorname{det} \nabla F(e)$.

Remark B.3. Starting with a domain with several holes, a similar construction as in Example B.1 with a subsequent orientation-preserving deformation can also cause the images of holes to be stacked inside of each other. In fact, this way, with $|n|$ holes for any given $n \in \mathbb{Z}$, we can get a deformation $y$ invertible on $\partial \Omega$, $\operatorname{such}$ that $\operatorname{deg}(y ; \Omega ; \cdot)$ attains the value $n$ somewhere. This also works in context of Example B.2 if we have several small conical holes that all meet at the tip of the big outer cone.

Acknowledgements. This research was supported by the Czech Science Foundation (GA CR) and the Austrian Science Fund (FWF) through the bilateral grant 19-29646L (Large Strain Challenges in Materials Science), and through the associated MSMT-WTZ bilateral travel grant 8J19AT013.

\section{REFERENCES}

[1] J. W. Alexander. An example of a simply connected surface bounding a region which is not simply connected. Proceedings of the National Academy of Sciences, 10(1):8-10, 1924. 
[2] J. W. Alexander. On the subdivision of 3-space by a polyhedron. Proceedings of the National Academy of Sciences of the United States of America, 10(1):6-8, 1924.

[3] J.M. Ball. Global invertibility of Sobolev functions and the interpenetration of matter. Proc. R. Soc. Edinb., Sect. A, Math., 88:315-328, 1981.

[4] John M. Ball. Convexity conditions and existence theorems in nonlinear elasticity. Arch. Rational Mech. Anal., 63(4):337-403, 1977.

[5] Marco Barchiesi, Duvan Henao, and Carlos Mora-Corral. Local invertibility in Sobolev spaces with applications to nematic elastomers and magnetoelasticity. Arch. Ration. Mech. Anal., 224(2):743-816, 2017.

[6] Ondřej Bouchala, Stanislav Hencl, and Anastasia Molchanova. Injectivity almost everywhere for weak limits of Sobolev homeomorphisms. Preprint arXiv:1912.05413, 2019.

[7] Morton Brown. Locally flat imbeddings of topological manifolds. Ann. of Math. (2), 75:331-341, 1962.

[8] Philippe G. Ciarlet and Jindřich Nečas. Injectivity and self-contact in nonlinear elasticity. Arch. Ration. Mech. Anal., 97:173-188, 1987.

[9] Sara Daneri and Aldo Pratelli. A planar bi-Lipschitz extension theorem. Adv. Calc. Var., 8(3):221-266, 2015.

[10] I. Fonseca and W. Gangbo. Local invertibility of Sobolev functions. SIAM J. Math. Anal., 26(2):280-304, 1995.

[11] Irene Fonseca and Wilfrid Gangbo. Degree theory in analysis and applications, volume 2 of Oxford Lecture Series in Mathematics and its Applications. The Clarendon Press, Oxford University Press, New York, 1995. Oxford Science Publications.

[12] Ralph H. Fox and Emil Artin. Some wild cells and spheres in three-dimensional space. Ann. of Math. (2), 49:979-990, 1948.

[13] Alessandro Giacomini and Marcello Ponsiglione. Non-interpenetration of matter for SBV deformations of hyperelastic brittle materials. Proc. Roy. Soc. Edinburgh Sect. A, 138(5):1019-1041, 2008.

[14] Jasun Gong. A Schoenflies extension theorem for a class of locally bi-Lipschitz homeomorphisms. Michigan Math. J., 60(3):507-523, 2011.

[15] Diego Grandi, Martin Kružík, Edoardo Mainini, and Ulisse Stefanelli. A phasefield approach to Eulerian interfacial energies. Arch. Ration. Mech. Anal., 234(1):351-373, 2019 .

[16] Duvan Henao and Carlos Mora-Corral. Invertibility and weak continuity of the determinant for the modelling of cavitation and fracture in nonlinear elasticity. Arch. Ration. Mech. Anal., 197(2):619-655, 2010.

[17] Stanislav Hencl and Pekka Koskela. Lectures on mappings of finite distortion, volume 2096 of Lecture Notes in Mathematics. Springer, Cham, 2014.

[18] Stanislav Hencl and Kai Rajala. Optimal assumptions for discreteness. Arch. Ration. Mech. Anal., 207(3):775-783, 2013.

[19] Tadeusz Iwaniec and Jani Onninen. Monotone Sobolev mappings of planar domains and surfaces. Arch. Ration. Mech. Anal., 219(1):159-181, 2016.

[20] Tadeusz Iwaniec and Jani Onninen. Radó-Kneser-Choquet theorem for simply connected domains ( $p$-harmonic setting). Trans. Amer. Math. Soc., 371(4):2307-2341, 2019.

[21] Agnieszka Kałamajska, Stefan Krömer, and Martin Kružík. Sequential weak continuity of null Lagrangians at the boundary. Calc. Var. Partial Differential Equations, 49(3-4):1263-1278, 2014. 
[22] Hansjörg Kielhöfer. Bifurcation theory, volume 156 of Applied Mathematical Sciences. Springer, New York, second edition, 2012. An introduction with applications to partial differential equations.

[23] Stefan Krömer and Jan Valdman. Global injectivity in second-gradient nonlinear elasticity and its approximation with penalty terms on the boundary. In preparation.

[24] Stefan Krömer and Jan Valdman. Global injectivity in second-gradient nonlinear elasticity and its approximation with penalty terms. Mathematics and Mechanics of Solids (online first), 2019.

[25] J. Luukkainen and J. Väisälä. Elements of Lipschitz topology. Ann. Acad. Sci. Fenn. Ser. A I Math., 3(1):85-122, 1977.

[26] M. Marcus and V. J. Mizel. Transformations by functions in Sobolev spaces and lower semicontinuity for parametric variational problems. Bull. Amer. Math. Soc., 79:790-795, 1973.

[27] O. Martio and William P. Ziemer. Lusin's condition (N) and mappings with nonnegative Jacobians. Michigan Math. J., 39(3):495-508, 1992.

[28] B. C. Mazur. On embeddings of spheres. Acta Math., 105:1-17, 1961.

[29] G. H. Meisters and C. Olech. Locally one-to-one mappings and a classical theorem on schlicht functions. Duke Math. J., 30:63-80, 1963.

[30] John Milnor. On manifolds homeomorphic to the 7-sphere. Ann. of Math. (2), 64:399-405, 1956.

[31] Anastasia Molchanova and Sergey Vodopyanov. Injectivity almost everywhere and mappings with finite distortion in nonlinear elasticity. Calculus of Variations and Partial Differential Equations, 59(1):17, 2019.

[32] Stefan Müller. Higher integrability of determinants and weak convergence in $L^{1}$. J. Reine Angew. Math., 412:20-34, 1990.

[33] Stefan Müller and Scott J. Spector. An existence theory for nonlinear elasticity that allows for cavitation. Arch. Rational Mech. Anal., 131(1):1-66, 1995.

[34] Stefan Müller, Scott J. Spector, and Qi Tang. Invertibility and a topological property of Sobolev maps. SIAM J. Math. Anal., 27(4):959-976, 1996.

[35] Enrique Outerelo and Jesús M. Ruiz. Mapping degree theory, volume 108 of Graduate Studies in Mathematics. American Mathematical Society, Providence, RI; Real Sociedad Matemática Española, Madrid, 2009.

[36] Kai Rajala. Remarks on the Iwaniec-Šverák conjecture. Indiana Univ. Math. J., 59(6):2027-2039, 2010.

[37] Kai Rajala. Reshetnyak's theorem and the inner distortion. Pure Appl. Math. Q., 7(2, Special Issue: In honor of Frederick W. Gehring, Part 2):411-424, 2011.

[38] Yu. G. Reshetnyak. Space mappings with bounded distortion, volume 73 of Translations of Mathematical Monographs. American Mathematical Society, Providence, RI, 1989. Translated from the Russian by H. H. McFaden.

[39] Qi Tang. Almost-everywhere injectivity in nonlinear elasticity. Proc. Roy. Soc. Edinburgh Sect. A, 109(1-2):79-95, 1988.

[40] C. J. Titus and G. S. Young. The extension of interiority, with some applications. Trans. Amer. Math. Soc., 103:329-340, 1962.

[41] Pekka Tukia. The planar Schönflies theorem for Lipschitz maps. Ann. Acad. Sci. Fenn. Ser. A I Math., 5(1):49-72, 1980.

[42] Enrique Villamor and Juan J. Manfredi. An extension of Reshetnyak's theorem. Indiana Univ. Math. J., 47(3):1131-1145, 1998.

[43] Sergey Vodopyanov and Vladimir Goldshtein. Quasiconformal mappings and spaces of functions with generalized first derivatives. Siberian Mathematical Journal - SIB MATH J-ENGL TR, 17:399-411, 1976. 
[44] Vladimír Šverák. Regularity properties of deformations with finite energy. Arch. Rational Mech. Anal., 100(2):105-127, 1988.

[45] Alan Weinstein. A global invertibility theorem for manifolds with boundary. Proc. Roy. Soc. Edinburgh Sect. A, 99(3-4):283-284, 1985.

[46] Gordon Thomas Whyburn. Topological analysis. Princeton Mathematical Series. No. 23. Princeton University Press, Princeton, N. J., 1958.

Stefan Krömer, The Czech Academy of Sciences, Institute of Information Theory and Automation, Pod vodárenskou věŽí 4, 18208 Praha 8, Czech Republic, E-mail address: skroemer@utia.cas.cz 